RADFLO Physics and Algorithms

Eugene M. D. Symbalisty

John Zinn

Rodney W. Whitaker 


\section{DISCLAIMER}

Portions of this document may be illegible in electronic image products. Images are produced from the best available original document. 


\section{TABLE OF CONTENTS}

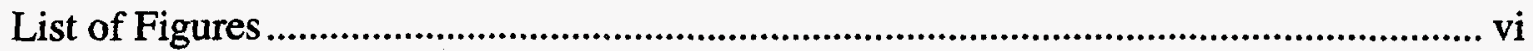

List of Tables ............................................................................................................ vii

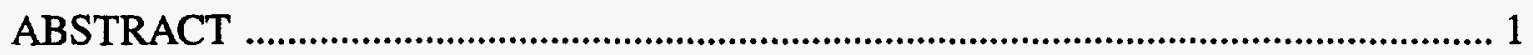

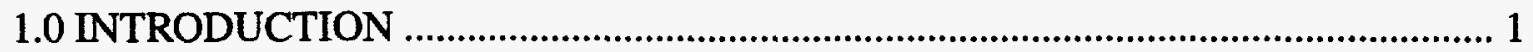

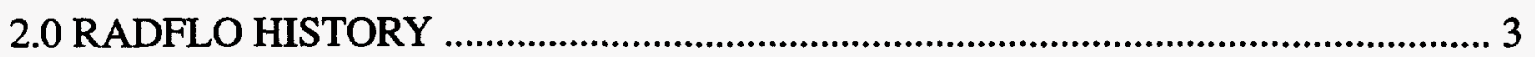

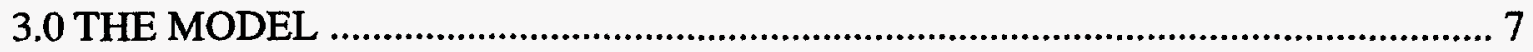

3.1 Air Equation of State and Absorption Coefficient ................................... 9

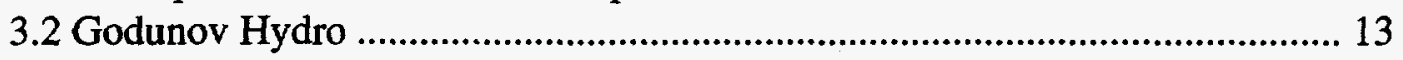

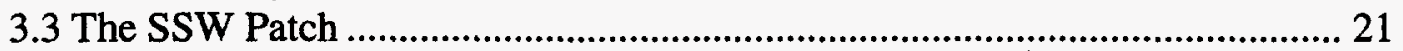

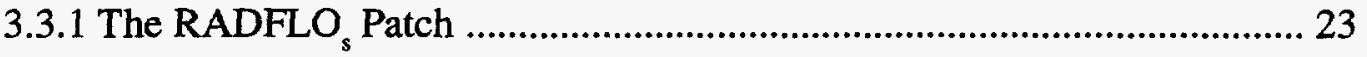

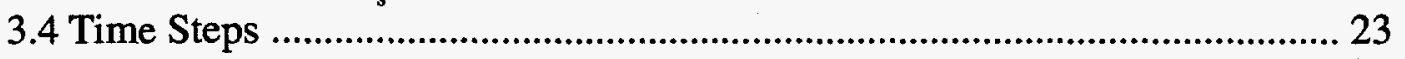

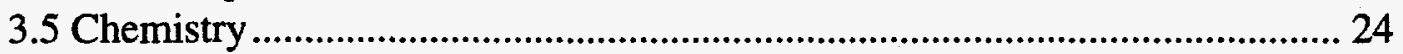

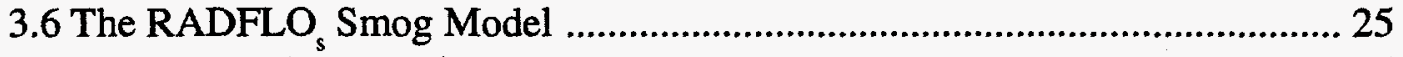

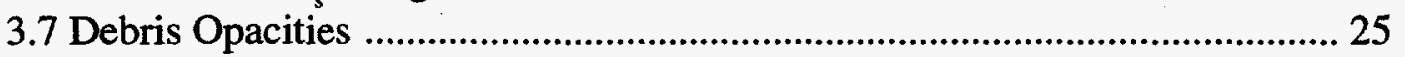

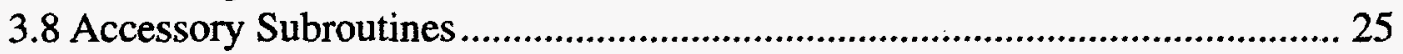

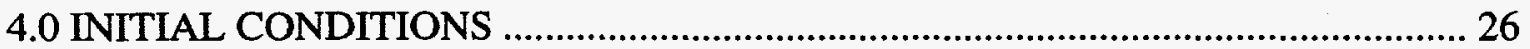

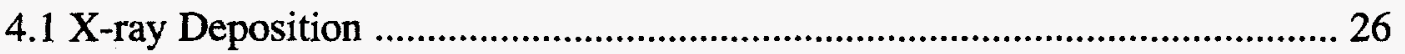

4.2 HYCHEM Input Parameters ................................................................... 27

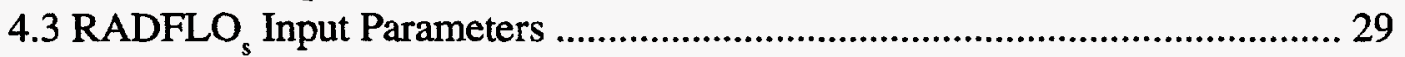

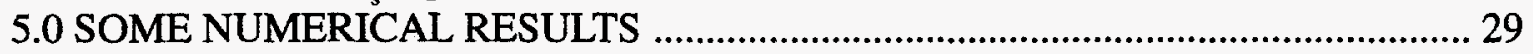

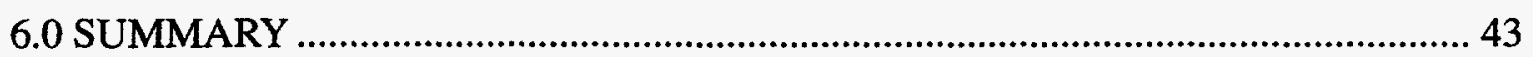

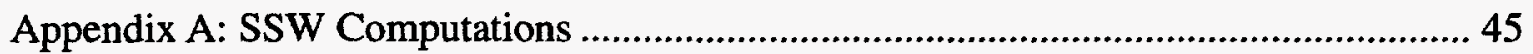

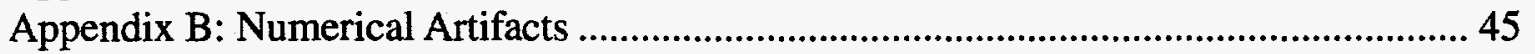

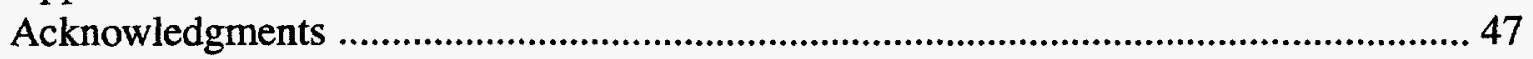

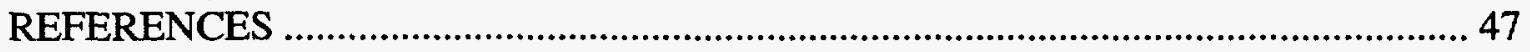




\section{LIST OF FIGURES}

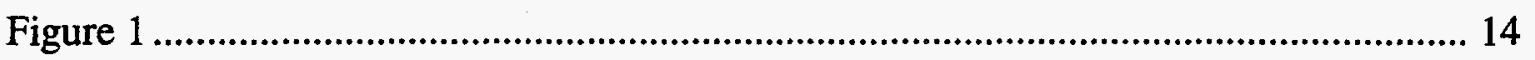

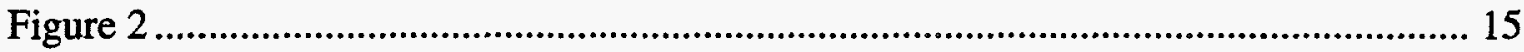

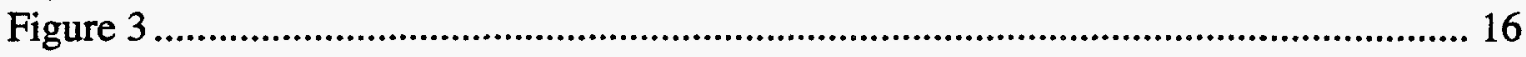

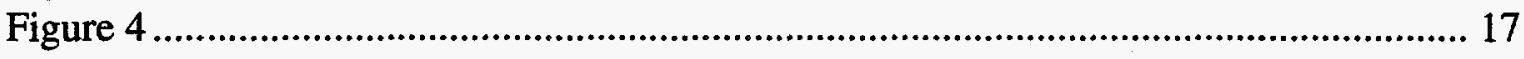

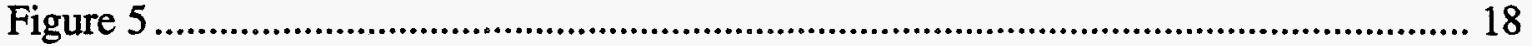

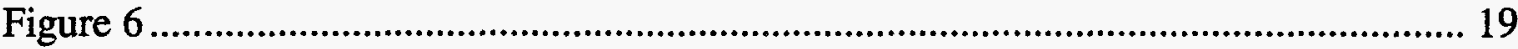

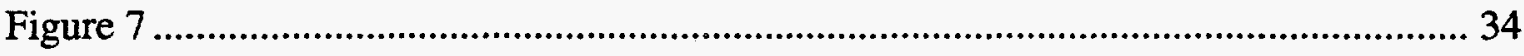

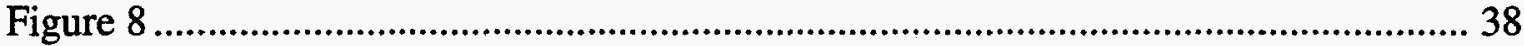

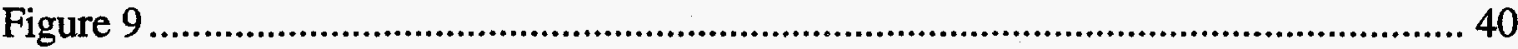

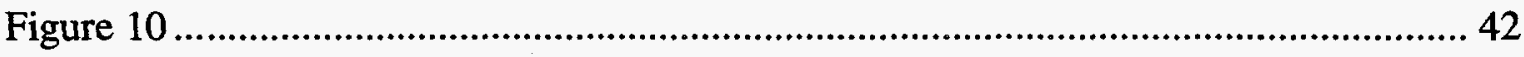

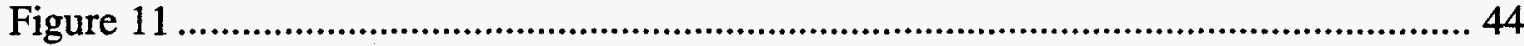

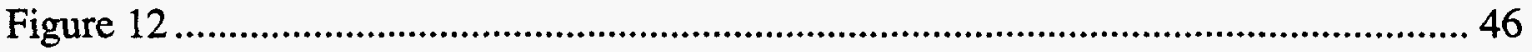




\section{LIST OF TABLES}

Table I: HYCHEM Input Variables 28

Table II: RADFLO Input Variables 30

Table III: More RADFLO Input Variables 31

Table IV: Computed Optical Power Results for Sea-Level Altitude Explosions 33

Table V: Computed Optical Power Results for High-Altitude Explosions 33 



\title{
RADFLO PHYSICS AND ALGORITHMS
}

by

Eugene M. D. Symbalisty, John Zinn, and Rodney W. Whitaker

\begin{abstract}
This paper describes the history, physics, and algorithms of the computer code RADFLO and its extension HYCHEM. RADFLO is a one-dimensional, radiation-transport hydrodynamics code that is used to compute early-time fireball behavior for low-altitude nuclear bursts. The primary use of the code is the prediction of optical signals produced by nuclear explosions. It has also been used to predict thermal and hydrodynamic effects that are used for vulnerability and lethality applications. Another closely related code, HYCHEM, is an extension of RADFLO which includes the effects of nonequilibrium chemistry. Some examples of numerical results will be shown, along with scaling expressions derived from those results. We describe new computations of the structures and luminosities of steady-state shock waves and radiative thermal waves, which have been extended to cover a range of ambient air densities for high-altitude applications. We also describe recent modifications of the codes to use a one-dimensional analog of the CAVEAT fluid-dynamics algorithm in place of the former standard Richtmyer-von Neumann algorithm.
\end{abstract}

\subsection{INTRODUCTION}

RADFLO is a spherically-symmetric, multifrequency, radiation-transport and Lagrangian hydrodynamics code designed for modeling nuclear fireballs in the atmosphere for altitudes up to about $50 \mathrm{~km}$. The model assumes local thermodynamic equilibrium and makes use of stored tables of the air equation of state (pressure, temperature, gamma, and sound speed as functions of density and internal energy) and of air opacity (vs density, temperature, and photon energy). A related code, HYCHEM, includes RADFLO but adds nonequilibrium chemistry and effects of gamma rays and neutrons as well.

As the code runs it generates profiles of temperature, density, material velocity, pressure, and brightness at various wavelengths-for a sequence of times. At the end of the run, RADFLO produces plots of radiated power vs time in several wavelength 
bands along with plots of fireball radius vs time and expansion velocity vs time. RADFLO is also used to provide initial conditions for related problems (Jones et al. 1987, Symbalisty et al. 1987, Symbalisty 1991).

The code is designed to treat the evolution of the air fireball as accurately as possible. The bomb itself and associated bomb debris are treated relatively crudely. The debris equation of state and opacities are taken to be the same as those of air with a few exceptions, which are described later in this report. Also, we do not attempt to compute the Taylorunstable debris expansion; the debris cloud radius is modeled with a crude empirical algorithm.

The altitude range of applicability of the code is limited mainly by the assumption of spherical symmetry. That is, the radiation transport and hydrodynamic motions must produce a spherical fireball approximately concentric with the bomb. This implies roughly that, for the dominant wavelengths of radiation emitted by the bomb, the mean free paths must be small compared to the atmospheric scale height. Also the fireball radius (during times of interest) must be smaller than the scale height. For a Megaton device this implies an altitude limit of about $50 \mathrm{~km}$; however, for a small yield device with few energetic $\mathrm{x}$-rays the limit can be higher. The term yield is the sum of the energy radiated from the case in $\mathrm{x}$ rays, the residual debris' internal energy, and the debris' kinetic energy.

A distinctive feature of RADFLO is the algorithm used to compute the radiation transport. Functionally it is a "two-stream" approximation, implying that for each frequency group the intensity function has one value for polar angles smaller than a prescribed angle $\theta_{s}$ and another value for $\theta \geq \theta_{s}$. The angle $\theta_{s}$ is computed at each radial location and time and corresponds to the angle subtended by the opaque "fireball." Inside the fireball itself, $\theta_{s}$ is set to $\pi / 2$. In regions that are optically thick, the diffusion approximation is used. The transport computation is done for each of 42 frequency groups ranging from the far infrared up through the soft $x$-ray region (Zinn 1973).

A bothersome feature of the physical and numerical problem is a very bad mismatch of length scales between the fireball itself, which has dimensions of meters to tens of meters, and the thickness of the fireball surface region that determines its brightness (i.e., a shock front or thermal-wave front), which is typically millimeters to centimeters. To cope with this problem a separate computer code, $\mathrm{SSW}$, computes the temperature structures and radiant fluxes in steady-state shock waves and thermal waves (i.e. waves that propagate with a time-invariant structure, such that the time can be eliminated from the radiation and hydrodynamic equations). The results of these steady-state computations are used 
within RADFLO for computing the radiation fluxes emanating from the fireball front at early times (for wavelengths longer than $180 \mathrm{~nm}$ and times when the front velocity exceeds $30 \mathrm{~km} / \mathrm{s}$ ).

Over the years a number of people have worked with RADFLO at Los Alamos National Laboratory (LANL) or Mission Research Corporation (MRC), and possibly other institutions. In this report, especially in the historical sections, the present authors use the term "we," which in some cases refers to these past participants. Thus "we" should be interpreted as the present authors and past contributors. In particular this report draws heavily on the description of RADFLO in four reports by Sappenfield (1973, 1974, 1975, 1976).

\subsection{RADFLO HISTORY}

The first version of RADFLO was written in 1963 shortly after the high-altitude nuclear test series of Operation Dominic in order to model and analyze the Bluegill event (at "intermediate" altitude). For that objective it was written initially as a radiation transportonly code without hydrodynamics (Zinn 1964). That initial version was fairly successful in matching the fireball optical output vs time to the end of the main optical pulse. Hydrodynamics was added to the code in 1964. With the hydrodynamics the Bluegill calculations were extended to later times (several seconds), and comparisons with the Bluegill measurements of brightness, radiated power, and fireball dimensions were amazingly good (Zinn and Fajen 1964; Zinn 1965).

During the following year RADFLO computations were run for the Tightrope event (somewhat smaller yield and lower in altitude). Based on comparisons of the computed results with Tightrope radius-time data, the estimated Tightrope yield was revised upward.

After an hiatus of five years due to shifts in military interest to higher altitude, RADFLO was again used in the early 1970s for the nuclear tests at sea level, in particular the French tests (Zinn et al. 1970). RADFLO did a good job of modeling a sea level explosion in the time regime from shortly after first maximum until shortly after second maximum, and was also useful for the early-time pre-first-maximum phase of large-yield explosions for studies of the x-ray veil (Zinn et al. 1972; Zinn, Kodis, and Sutherland 1974). However, for the time span between veil breakthrough and first maximum the computed brightness and radiated power results exhibited very large oscillations. These oscillations could be attributed to the necessarily coarse spatial mesh used in the code, whose dimensions were several orders of magnitude larger than those expected of the fireball front (i.e. a shock front or radiative thermal-wave front). 
Since the detailed temperature structure of the front determines its brightness, we embarked on a separate study of the structure of high-velocity shock waves which included radiation effects. For this study we transformed the radiation and hydrodynamic equations into a coordinate system moving at the shock velocity, with the assumption that in the moving coordinate system the temperature and density structure would be time-invariant. The finite difference representations of these equations were incorporated into a code called SSS (steady-state shock). The results showed that for shock waves with velocities in the range 30 to $80 \mathrm{~km} / \mathrm{s}$, the brightness-determining structure had dimensions of a millimeter or so, and the effective brightness temperature for velocities above $30 \mathrm{~km} / \mathrm{s}$ was significantly smaller than the shock Rankine-Hugoniot temperature. Above $80 \mathrm{~km} / \mathrm{s}$ there was an apparent breakdown of the steady-state assumption. The SSS results were published (Zinn and Anderson 1973) but not incorporated into RADFLO until several years later.

In the mid-1970s several projects compared RADFLO computations with old US nuclear test data and with data from the French tests (e.g. Horak et al. 1983). We studied relationships between $\mathrm{x}$-ray veil breakthrough times and expected nuclear device $\mathrm{x}$-ray outputs (Zinn et al. 1970), and we compared fireball outputs at X- and K-band microwave wavelengths with microwave radiometer data (Zinn et al. 1974). We looked at the contribution due to device neutrons and gamma rays of air fluorescence to the total optical power vs time. We made detailed comparisons of computed minimum and second maximum times with yield-scaling laws derived from nuclear test data (Zinn et al. 1972; Zinn, Kodis, and Sutherland 1974). We commenced studies on the effects of nuclear "smog" associated with nonequilibrium chemistry $\left(\mathrm{NO}_{2}\right.$, ozone, etc.) outside the fireball. Also during the 1970 s several two-dimensional late-time fireball codes were developed which used RADFLO results as input.

Also in the 1970 s the code began to proliferate into several separate versions. In 1971 the then-current code was taken to MRC, where RADFLO was modified for a smaller computer and subsequently further modified in other ways. The name RADFLO was retained. The MRC RADFLO code was used extensively for analysis of satellite bhangmeter data and for Defense Nuclear Agency (DNA) systems studies (Sappenfield 1973, 1974, 1975, and 1976). The MRC code was supplied under contract to the Air Force. Also, a reorganization within Los Alamos led to two separate RADFLO versions in two groups, which gradually diverged from one another. For the version upon which this report is based, modifications and improvements made during the 1970s were usually discussed with MRC, 
so there are strong resemblances between this code and the MRC version. In this period we became interested in nonequilibrium chemistry effects and "smog," and we continued to look for solutions to the first-maximum radiant-power oscillation problem.

Only a few comments will be made on the second Los Alamos version of the RADFLO code mentioned in the paragraph above. This version was used for low-altitude $(<30 \mathrm{~km})$ bursts and assumes equilibrium chemistry in the equation of state. It has been used for prediction of power-time curves and airblast effects, as well as initialization of two-dimensional calculations. Documentation can be found in Horak and Kodis (1983). The appendix of the Horak and Kodis report describes the use of fine zoning to achieve overall $3 \%$ accuracy in shock over pressures using initial zoning of $2.5 \mathrm{~cm} / \mathrm{kt}^{1 / 3}$. The report also includes a description of the use of discrete ordinate radiation-transport algorithm. This version has been used to calculate the evolution of concentric nuclear explosions; bursts at the same place but separated in time (Jones et al. 1983).

Another area of interest has been the study of mass effects on optical-power time histories. The original RADFLO essentially assumed a point explosion with a very high yield to mass ratio. Realistic mass values were modeled with a heavy-air approximation (Horak 1980; Jones et al. 1980). This approximation assumes that the mass of the device or the mass immediately surrounding the device can be modeled, to first order, as high-density or heavy air. Extreme effects of shielding can be found in Symbalisty (1994) where a different material, with its own equation of state and opacity, is used to model the mass surrounding the device. The key result of large amounts of mass surrounding the device is the distortion of the power-time curve up to and around first maximum. This is the time regime where RADFLO is least equipped to give reliable answers due to the oscillation problem and the simple device description.

From 1979 to 1982 we extended the steady-state shock wave model to include radiation transport-dominated waves (thermal waves), which led to a steady-state thermalwave computer model, SST, with strong similarities to the SSS model. The two computer codes were merged into a single steady-state shock and thermal-wave code, SSW (Zinn and Sutherland 1981). The results showed that for both "shock waves" (i.e. waves dominated by hydrodynamics-with propagation velocities below $80 \mathrm{~km} / \mathrm{s}$ ) and "thermal waves" (i.e. waves dominated by radiation transport-with velocities above $80 \mathrm{~km} / \mathrm{s}$ ) the thickness of the visible surface layer was a centimeter or less, and the effective visible brightness temperature was much smaller than the actual temperature behind the wave front. The computed luminosity 
results could be summarized in the form of plots of effective brightness temperature (for specified wavelength bands) vs propagation velocity, for given values of the ambient air density. Very recently (1995) we have extended the SSW computations to include a range of values of the background air density so that the results now apply to high altitudes as well as to sea level. These results are summarized in Appendix A of this report.

The SSW results showed that the thickness of the fireball radiating-surface layer was much smaller than the smallest possible zone thicknesses that could be used in a functioning explicit Lagrangian fireball rad-hydro code. Therefore we needed to devise a way of incorporating the results of the SSW computations into the RADFLO radiation transport algorithm. The best approach was to compute the radiant intensity emanating from the fireball surface from the computed velocity of that surface, using a stored tabulation of brightness temperature vs velocity. The resulting RADFLO was free of most of the troublesome oscillations (Zinn and Sutherland 1982). However, persistent nagging questions remained concerning precisely where to define the fireball front, how to compute its velocity, and how to correct for the effects of the artificial viscous pressure.

As noted earlier, the LANL (Zinn, Kodis, and Sutherland) and MRC (Sappenfield and McCartor) versions of RADFLO began to diverge in 1971. The latter version was brought back to LANL by D. Sappenfield in 1980 (Sappenfield 1983, 1984, and 1985), and since that time both codes have been maintained separately. This leads to some confusion which we have not quite sorted out. For now we will refer to the two versions as RADFLOz and RADFLO $_{S}$, respectively. A vintage 1979 version of $R A D F L O_{Z}$ was used for the Vela Alert747 computations and later described in a RADFLO User's Manual (Horak 1980; Jones et al. 1987; Horak and Kodis 1983). A more modern version of $\mathrm{RADFLO}_{\mathrm{Z}}$ is embodied within the code HYCHEM, which includes the nonequilibrium chemistry. RADFLO $\mathrm{Z}_{\mathrm{Z}}$ and HYCHEM have been modified and improved extensively during the past year.

Further code improvements and extensions in the 1980s included the development of HYCHEM. HYCHEM is an extension of RADFLO that includes computations of gamma ray and neutron-produced ionization rates with associated nonequilibrium chemistry processes and uses the computed chemical species concentrations in computing the radiative absorption coefficients, thus fully coupling the chemistry and radiation transport computations. As a test of HYCHEM, the 1956 Redwing-Navajo chord experiment was modeled, including time-resolved spectroscopic measurements of the gamma ray and neutronproduced smog outside of a fireball (Zinn et al. 1980, 1982). Several related studies validated the chemistry. These included development of a Teller light model in the TELLER and 
SLEMP computer codes, which were used to model the time-resolved Teller lightspectroscopy experiments in the Redwing-Dakota event (Zinn and Sutherland 1987).

Other code modifications in the late 1980s included development of a version with a noniterative Godunov-Riemann equation-solver hydrodynamics subroutine in place of the explicit Richtmyer-von Neumann hydrodynamics algorithm of previous versions (von Neumann and Richtmyer 1950). Also, a multimaterial RADFLO version (Symbalisty 1994) treats the bomb materials and possible surrounding solid or liquid layers as distinct from the surrounding air (i.e. with distinct equations of state and opacities). In addition, a research study was undertaken to incorporate effects of Taylor instabilities in the bomb debris expansion (Jones and Kodis 1986; Jones et al. 1979, 1981) but was discontinued.

Some further history relating to equations of state and absorption coefficients is included in section 3.1 .

\subsection{THE MODEL}

We list here the radiation-hydrodynamic equations that the RADFLO code solves (Zinn 1973) in spherical symmetry. The equations of conservation of mass, momentum and energy are

$$
\begin{aligned}
& \frac{\partial \rho}{\partial \tau}=-\nabla \cdot(\rho \mathbf{v}) \\
& \frac{\partial\left(\rho v_{\alpha}\right)}{\partial t}=-\nabla \cdot\left(\rho v_{\alpha} \mathbf{v}\right)-\nabla_{\alpha} P \\
& \frac{\partial e}{\partial t}=-\nabla \cdot(e v)-P \nabla \cdot \mathrm{v}-\int_{0}^{\infty} \nabla \cdot \mathrm{F}_{v} d v
\end{aligned}
$$

where the monochromatic flux function is

$$
\mathrm{F}_{v}=2 \pi \int_{0}^{\pi} I_{v}(\theta) \sin \theta \cos \theta d \theta
$$


and the specific monochromatic intensity at frequency $v$ is

$$
I_{v}\left(P_{2}\right)=I_{v}\left(P_{1}\right) \exp \left[-\int_{P_{1}}^{P_{2}} \mu^{\prime}(\xi) d \xi\right]+\int_{P_{1}}^{P_{2}} \mu^{\prime}(\xi) B_{v}(\xi) \exp \left[-\int_{\xi}^{P_{2}} \mu^{\prime}\left(\xi^{\prime}\right) d \xi^{\prime}\right] d \xi
$$

$P_{1}$ and $P_{2}$ are two points on the surface of a volume element. The angle $\theta$ is the angle between the radius vector and the direction of a specified ray. The differentials $d \xi$ and $d \xi^{\prime}$ are elements of distance along the ray connecting points $P_{1}$ and $P_{2} . B_{v}$ is the Planck function and $\mu^{\prime}$ is the linear absorption coefficient corrected for stimulated emission.

The pressure, $P$, and temperature, $T$, are assumed to be known functions of the mass density, $\rho$, and the material internal energy density, $e$ :

$$
P=P(\rho, e) \quad, \quad T=T(\rho, e)
$$

The radiative absorption coefficients are also assumed to be known:

$\mu^{\prime}=\mu^{\prime}(\rho, e, v)$.

Note that the force of gravity is not included in these equations, so that buoyant deformation of the fireball is not considered (nor are other two-dimensional effects). This implies a limit to the interval of time for reliable results. However, for low-altitude explosions this interval generally encompasses the time during which the main fireball radiation is emitted, unless there are significant ground interactions.

The basic radiation transport algorithm used in RADFLO is unchanged from that described in (Zinn 1973), except for two details which will be described below. The Richtmyer-von Neumann hydrodynamics algorithm is also unchanged. However, we have added the Godunov hydrodynamics subroutine which we now use as the standard. The older Richtmyer-von Neumann hydro-subroutine is still retained in the code for optional use. The set of air equation of state and opacity tables is unchanged except for a few minor changes in the opacities. The bomb initialization algorithm is also basically the same, although we now allow for an arbitrary number of mesh cells within the bomb volume. A large amount of new code was added in 1981 for patching in the SSW results, and these sections have been modified further during 1994-1995. 


\subsection{Air Equation of State and Absorption Coefficients}

The RADFLO, HYCHEM, and SSW codes all contain built-in tables of Planck functions and air equations of state and opacities. (The opacity $\kappa^{\prime}\left[\mathrm{cm}^{2} / \mathrm{g}\right]$ is equal to the linear absorption coefficient $\mu^{\prime}\left(\mathrm{cm}^{-1}\right)$ divided by the density $\rho$-both at specified frequency $v$ and corrected for stimulated emission).

The equation of state tables are used to find the cell values of the pressure $P$ and temperature $T$ from the corresponding computed cell values of $\rho$ and $E$. For ease and accuracy of interpolation the equation of state data are stored in the form of $P / \rho E$ and $T / E$ vs $E$ and $\rho$, using evenly-spaced logarithmic arrays of $E$ (90 values from $2 \times 10^{9}$ to $1 \times 10^{16} \mathrm{erg} / \mathrm{g}$ ) and of $\rho$ (eight values from $1.29 \times 10^{-9}$ to $1.29 \times 10^{-2} \mathrm{~g} / \mathrm{cm}^{3}$ ). Since the logarithmic spacings in both arrays are fixed, the interpolations can be very rapid, since for given values of a local cell energy and density the interpolation parameters can be calculated directly, without table searching. The quantities $P / \rho E$ and $T / E$ are relatively slowly varying functions of $\rho$ and $E$, so that interpolation of those quantities instead of just $P$ and $T$ can be carried out with relatively little error. When $P / \rho E$ and $T / E$ have been interpolated, the corresponding $P$ and $T$ can be immediately calculated.

The opacities are stored in a three-dimensional array as functions of $\rho, T$, and $h v$. The same evenly-spaced logarithmic array of $\rho$ is used, together with an evenly-spaced log array of $T$ values and an evenly-spaced array of $h v$ values. Interpolation of opacities, $\boldsymbol{\kappa}^{\prime}$, instead of $\mu^{\prime}$ minimizes interpolation errors, since $\kappa^{\prime}$ varies more gradually with density than does $\mu^{\prime}$, and as in the case of the equation of state data above, the interpolation indices can be calculated directly without table searches. With respect to the quantum energy variable $h v$, no interpolations are needed since the radiation transport computation is carried out for each of the same 42 groups as those of the opacity tabulation. Once the interpolated values of $\kappa^{\prime}$ are obtained, the absorption coefficients can be immediately calculated from $\mu^{\prime}=\rho \kappa^{\prime}$.

For each of the logarithmically-spaced values of $h v$ in the tabulations there corresponds a quantum energy interval $\delta h v$. For each $T$ and $h v$ we also tabulate a precomputed value of the Planck function integral $\int_{h v_{i i}}^{h v_{2 i}} B_{v} d v$, where $h v_{1 i}$ and $h v_{2 i}$ are the values of $h v$ at the two ends of the interval $i$. In carrying out the transport computation, when Planck function integrals are required we interpolate them from the tables in the same manner as the opacities. This is faster computationally than computing the Planck integrals on the fly.

The same interpolation scheme and the same tabular arrays of $\rho, E$, and $h v$ have 
been used in all versions of RADFLO and HYCHEM since 1965. Also the same equation of state tables are used. However, between the $\mathrm{RADFLO}_{\mathrm{Z}}$ and $\mathrm{RADFLO}_{\mathrm{S}}$ versions there are some small differences in the opacity tables.

With respect to development of the opacity data, gaps exist in the history due to poor documentation. Intensive research efforts to produce high-temperature air equation of state data (and computations) began during the Manhattan Project (Fuchs et al. 1942), and these efforts increased through the mid-1960s. In the 1950s and 1960s, most of this work was funded by the U.S. Air Force through the Air Force Special Weapons Command (AFSWC) and later the Air Force Weapons Laboratory (AFWL) and was carried out primarily at the RAND Corporation (Gilmore and Latter 1956; Gilmore 1955 and 1958; Latter 1954), and the National Bureau of Standards (Hilsenrath, Green, and Beckett 1956; Hilsenrath, Klein, and Woolley 1959; Woolley 1962). The equation of state was fairly well defined by the time of the initial development of RADFLO in 1963.

Research to produce reliable air-opacity data extended from the 1940s through the mid 1970s. Considerable work to assemble opacity tables for use in a fireball radiation transport code was done by A. Skumanich (1960) with help from H.A. Bethe. A large part of the opacity data came from Gilmore and Latter (1956). The first RADFLO code borrowed heavily from Skumanich's work. The first RADFLO opacity tables included only continuum absorption processes including free-free and bound-free transitions from atoms and ions of nitrogen, oxygen, and argon. No molecular bands or atomic lines were included. Below the atomic oxygen $L$ edge, the bound-free absorption coefficients for hot air were estimated using the Raizer approximation and with advice from H.A. Bethe.

From the 1950s through the 1970s, research on opacities was supported by AFSWC, AFWL, Los Alamos Scientific Laboratory, the Air Force Office of Scientific Research, Naval Research Laboratory, Defense Atomic Support Agency, and DNA. Experimental and theoretical work was carried out at RAND Corporation (Gilmore and Latter 1956; Gilmore 1958), Lockheed (Churchill, Hagstrom, and Landshoff 1964; Churchill et al. 1966; Buttrey and McChesney 1965; Mueller 1963; Johnston and Platas 1969; Armstrong et al. 1958; Churchill and Hagstrom 1964; Churchill et al. 1962; Johnston et al. 1971), AVCO Corporation (Allen et al. 1962; Keck et al. 1963; Taylor 1960; Taylor and Caledonia 1968; Wray and Taylor 1969; Keck et al. 1959; Kivel and Bailey 1957; Camm et al. 1967; Wentink et al. 1967;), Los Alamos Scientific Laboratory (Mayer 1947; Cox and Stewart 1964), AFWL (Harris and Sullo 1970; Generosa and Harris 1970; and Harris et al. 1969), General Electric 
Corporation (Breene 1966), California Institute of Technology (Patch et al. 1962), and elsewhere. Funding for opacity work tapered off sharply in the 1970s.

For the original RADFLO code, which included only continuum contributors to the opacity, 42 frequency groups were considered to be sufficient to resolve the most important structure in the spectra. However, as time went on, it became clear that accuracy would be improved greatly if molecular band systems and atomic lines were included. The fine spectral structures of these band systems and lines could not be resolved with 42 frequency groups so it was necessary to decide how to average the spectral details over each of the frequency groups. For spectral regions, temperatures, densities, and mesh cell thicknesses for which a cell was optically thick, a reasonable procedure was to use a "Rosseland" average of the absorption coefficients over each of the frequency groups. The Rosseland average tends to weight the valleys between the spectral lines. On the other hand, for conditions in which a cell is optically thin and where emission is dominant over absorption, generally it was better to use a "Planck" mean, which approximates a direct average of the absorption coefficient over the given frequency group.

In the late 1960s opacity groups were formed in the Los Alamos Theoretical Division and at AFWL. They maintained a close collaboration. The AFWL group produced a computer code known as ABSCO, which assembles atomic and molecular line atlases for given conditions of temperature and density (thermodynamic equilibrium assumed). The Los Alamos group produced a code that forms specified types of spectral averages of the opacities over specified frequency groups. This provided the opacity tables that we currently use in $\mathrm{RADFLO}_{Z}$ (and HYCHEM). These opacities are Rosseland averages over each of the specified 42 frequency groups that we use. The atomic, ionic, and molecular processes included in this opacity set are

- Free-free absorption in the fields of ions and neutrals, given the prevailing equilibrium populations of each.

- Bound-free continua for $\mathrm{N}$ atoms and ions, corresponding to the prevailing ionization levels and quantum state populations.

- Bound-free continua for $\mathrm{O}$ atoms and ions, corresponding to the prevailing ionization levels and quantum state populations.

- Bound-free continua for Argon atoms and ions, corresponding to the prevailing ionization levels and quantum state populations.

- Bound-bound transitions of $\mathrm{N}$ and $\mathrm{O}$. 
- Photodetachment from $\mathrm{O}^{-}$and $\mathrm{O}_{2}^{-}$.

- Photoionization of $\mathrm{N}_{2}$ and $\mathrm{O}_{2}$.

- $\mathrm{O}_{2}$ Schumann-Runge continuum.

- $\mathrm{O}_{2}$ photodissociation continuum number 2 .

- $\mathrm{O}_{2}$ Schumann-Runge band system.

- $\quad \mathrm{N}_{2}^{+}$band systems (9).

- $\quad \mathrm{N}_{2}$ band systems (4).

- $\quad$ NO band systems (11).

- $\mathrm{NO}^{+}$band system (1).

- $\quad$ CO band system (1).

- $\quad \mathrm{NO}_{2}$ bands.

- $\mathrm{CO}_{2} \mathrm{UV}$ band.

- $\quad \mathrm{CN}$ band systems (3).

Except for addition of the CO absorption the RADFLO opacities have not been modified since 1974.

Minor differences in the opacities used in RADFLO $\mathrm{S}_{\mathrm{S}}$ are described in Sowle et al. (1972) and Sappenfield (1973). In particular, the RADFLO $\mathrm{S}_{\mathrm{S}}$ opacities are $30 \%$ to $50 \%$ smaller than the $\mathrm{RADFLO}_{\mathrm{Z}}$ opacities in the temperature-wavelength regime that determines the optical power minimum time (i.e. $k T=0.2$ to $0.6 \mathrm{eV}$ and $h v=1.5$ to $4 \mathrm{eV}$ ). In this range the absorption is mainly due to $\mathrm{NO}_{2}$.

In Figures $1-4$ we plot the Rosseland and Planck mean absorption coefficients for eight different air temperatures as a function of photon energy. In $\mathrm{RADFLO}_{\mathrm{s}}$, the Rosseland mean is used if the optical depth, computed with the Rosseland values, is greater than one. The Planck mean is used if the optical depth, computed with the Planck values, is less than one, and a geometric average is used otherwise. The Planck mean absorption coefficient is always greater than or equal to the Rosseland mean.

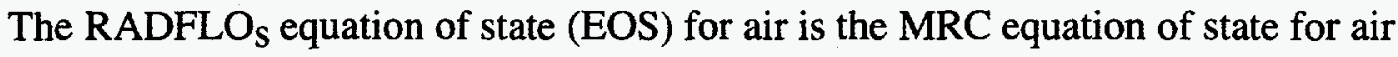
developed by Sappenfield and is very similar to the $\mathrm{RADFLO}_{Z}$ EOS. It relates the density $(\rho)$, the internal energy $(e)$, the temperature $(T)$, and pressure $(P)$. It is based on equation of state tables of Hilsenrath et al. $(1956,1959)$ and Gilmore $(1955)$. The temperature range of validity of the tables is $1000 \mathrm{~K}$ to $5 \times 10^{6} \mathrm{~K}$. Extrapolation beyond this range is straightforward. In terms of density the tables are valid for $1.29 \times 10^{-9} \leq \rho \leq 1.29 \times 10^{-2} \mathrm{gm} /$ cc.

The EOS will return $(T, \gamma-1)$ when given the state variables $(\rho, e)$ (Figures 5 and 
6). The pressure is then $p=(\gamma-1) \rho e$. The EOS will also return $(\gamma-1, e)$ when given $(T, \rho)$.

Godunov's method in the hydrodynamic solver requires two more parameters derived from ideal gas relations and the equation of state, the sound speed, $c_{s}$, and a shock parameter, $r a$. These parameters are defined as follows:

$c_{s}^{2}=\gamma P / \rho$, and $r a=1+(\gamma-1) / 2$.

\subsection{Godunov Hydro}

The staggered-mesh, Richtmyer-von Neumann hydrodynamics algorithm has been replaced with a variation of the Godunov method and uses an approximate Riemann solver (Dukowicz 1985). In fact, we are using a one-dimensional, spherically symmetric analog of the two-dimensional CAVEAT code developed at Los Alamos (Addessio et al. 1992). We quote from Addessio et al. (p. 7):

The benefits of a Godunov approach are of considerable importance. Staggeredmesh techniques result in a close coupling between pressure and velocity fields but usually require artificial dissipation for numerical stability and/or the achievement of proper entropy changes. Such artifical dissipation may be explicit (artifical viscosity) or hidden (donor-cell advection), but such methods typically fail to overcome mesh-drifting tendencies and are often excessively diffusive. With the Godunov approach, cell-edge pressures and velocities required for acceleration and work terms are obtained by solving the Riemann problem each cycle from the cellcentered state variables on the two sides of each cell edge. The result is again close coupling between pressure and velocity fields, usually with the optimal local level of dissipation. Because the minimal amount of dissipation is added by the Riemann solution, the Godunov method results in lower overall diffusion, and this does not mask the effect of realistic sources of diffusion from physical processes. 


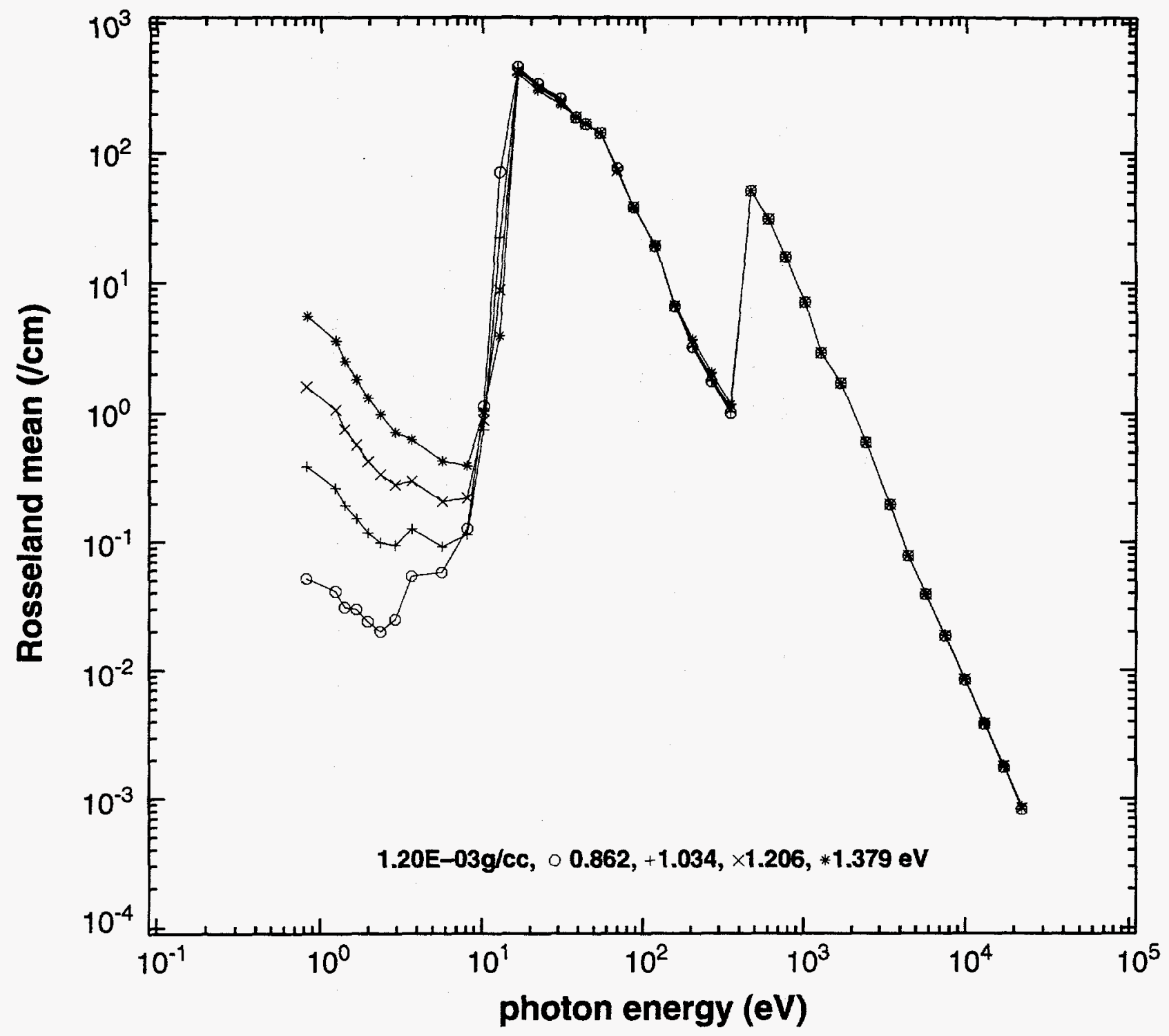

Figure 1. The Rosseland mean absorption coefficient as a function of photon energy for four different temperatures from the $\mathrm{RADFLO}_{s}$ tables. The air temperatures, in $\mathrm{eV}$, are $0.862,1.034,1.206$, and 1.379. 


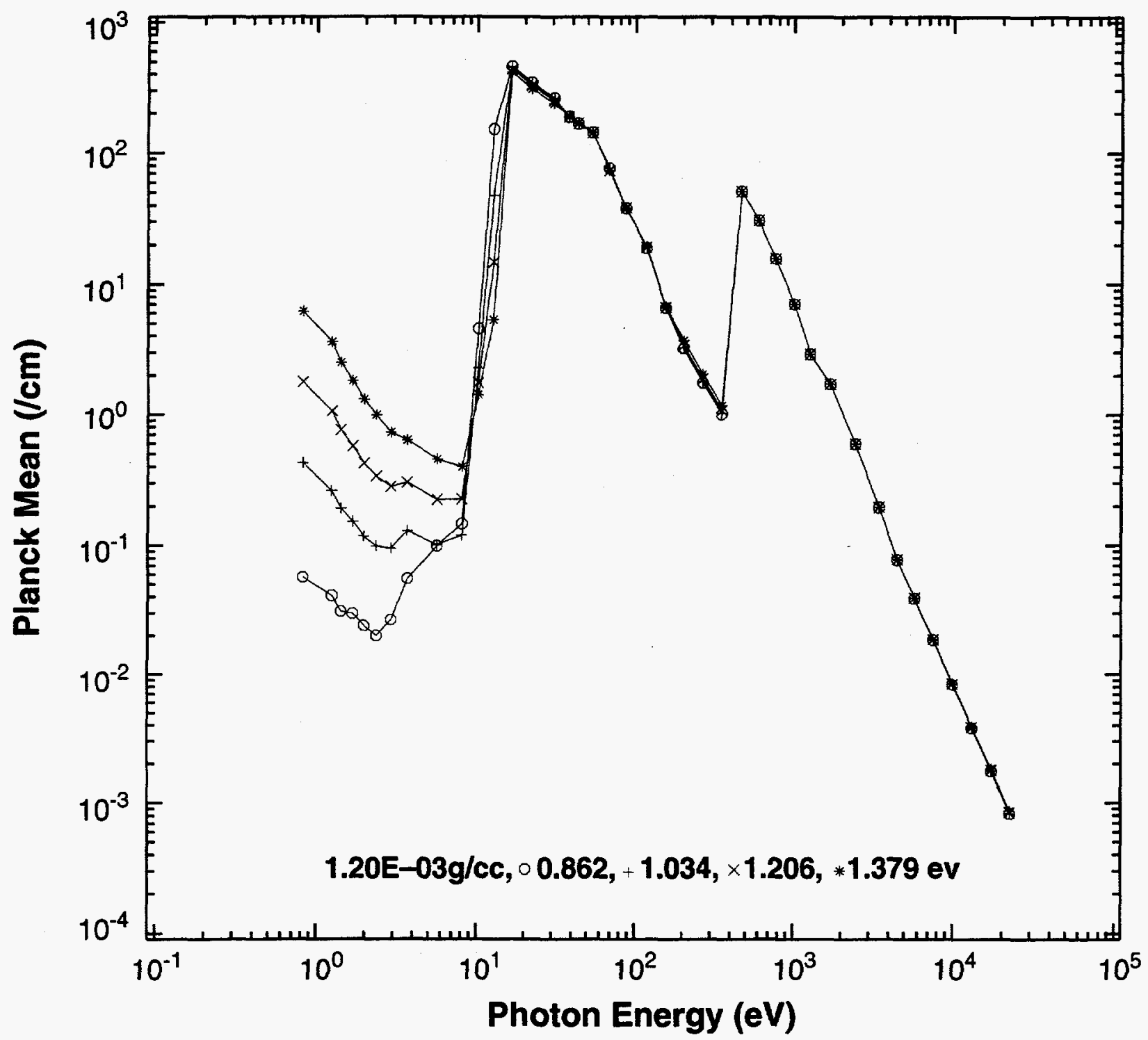

Figure 2. The Planck mean absorption coefficient as a function of photon energy for four different temperatures from the $\mathrm{RADFLO}_{\mathrm{s}}$ tables. The air temperatures, in $\mathrm{eV}$, are $0.862,1.034,1.206$, and 1.379 , which are the same values as the previous figure. 


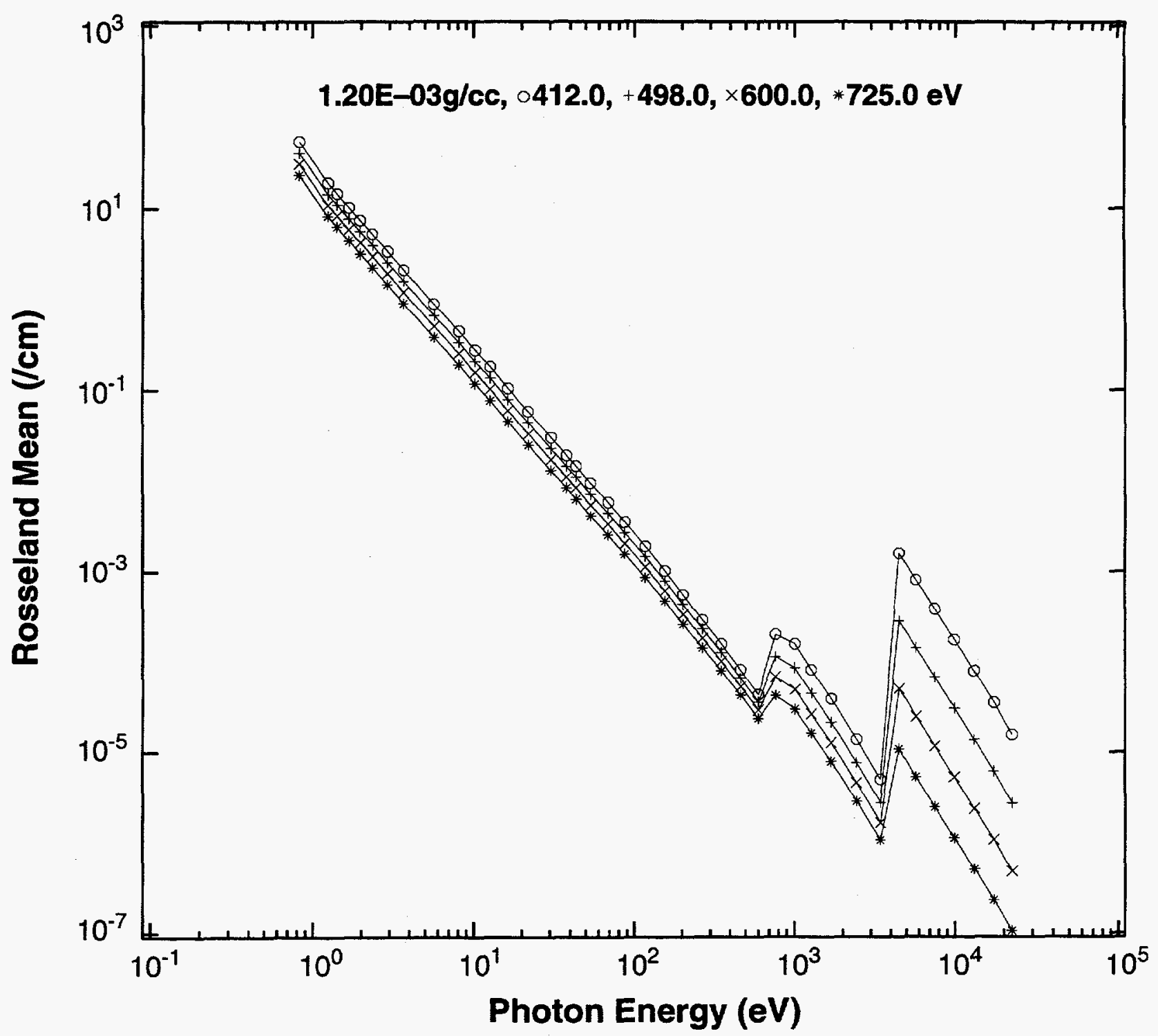

Figure 3. The Rosseland mean absorption coefficient as a function of photon energy for four high temperatures from the $\mathrm{RADFLO}_{\mathrm{S}}$ tables. The air temperatures, in $\mathrm{eV}$, are $412,498,600$, and 725 . 


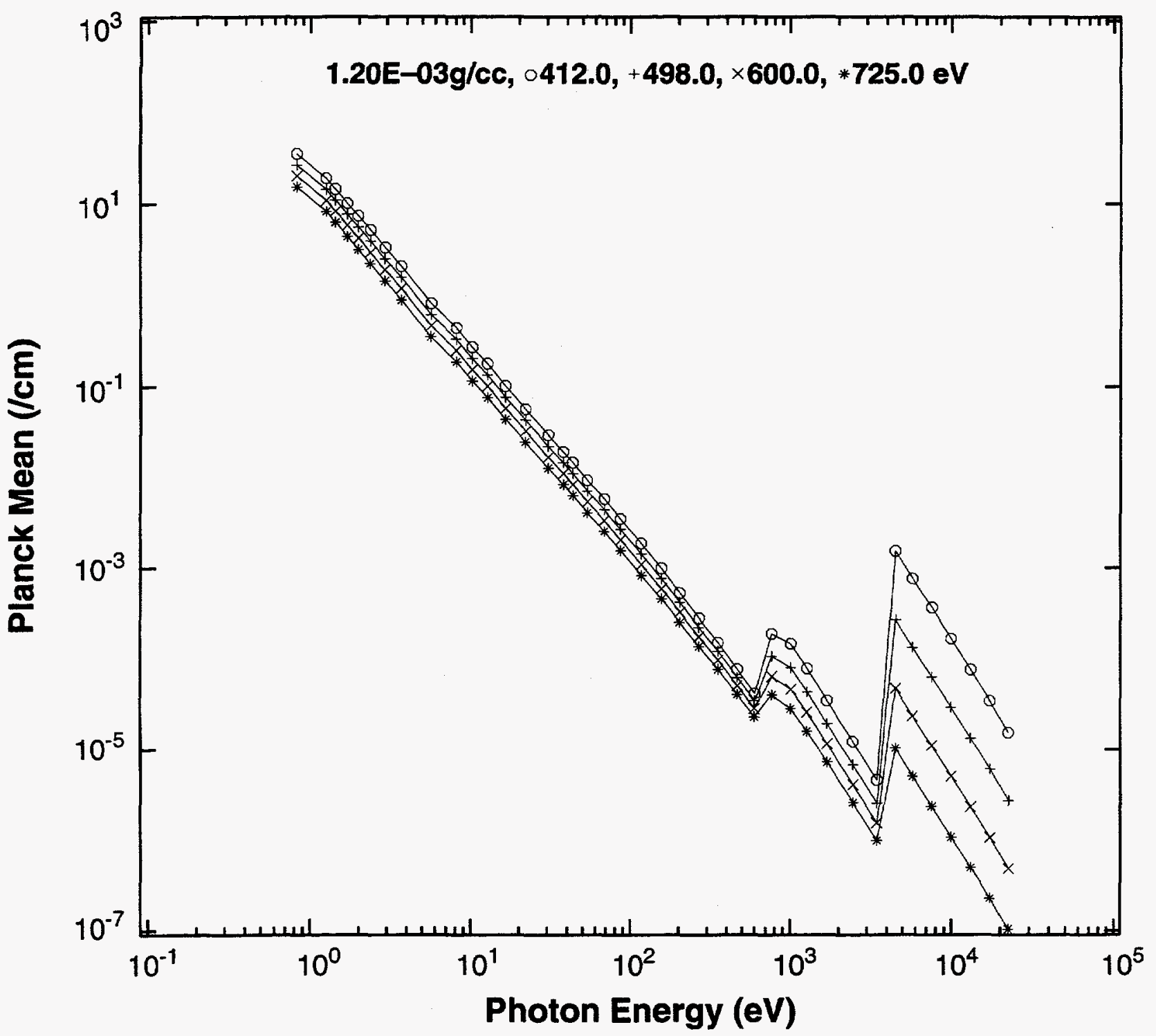

Figure 4. The Planck mean absorption coefficient as a function of photon energy for four high temperatures from the $\mathrm{RADFLO}_{\mathrm{s}}$ tables. The air temperatures, in $\mathrm{eV}$, are $412,498,600$, and 725 , which are the same values as the previous figure. 


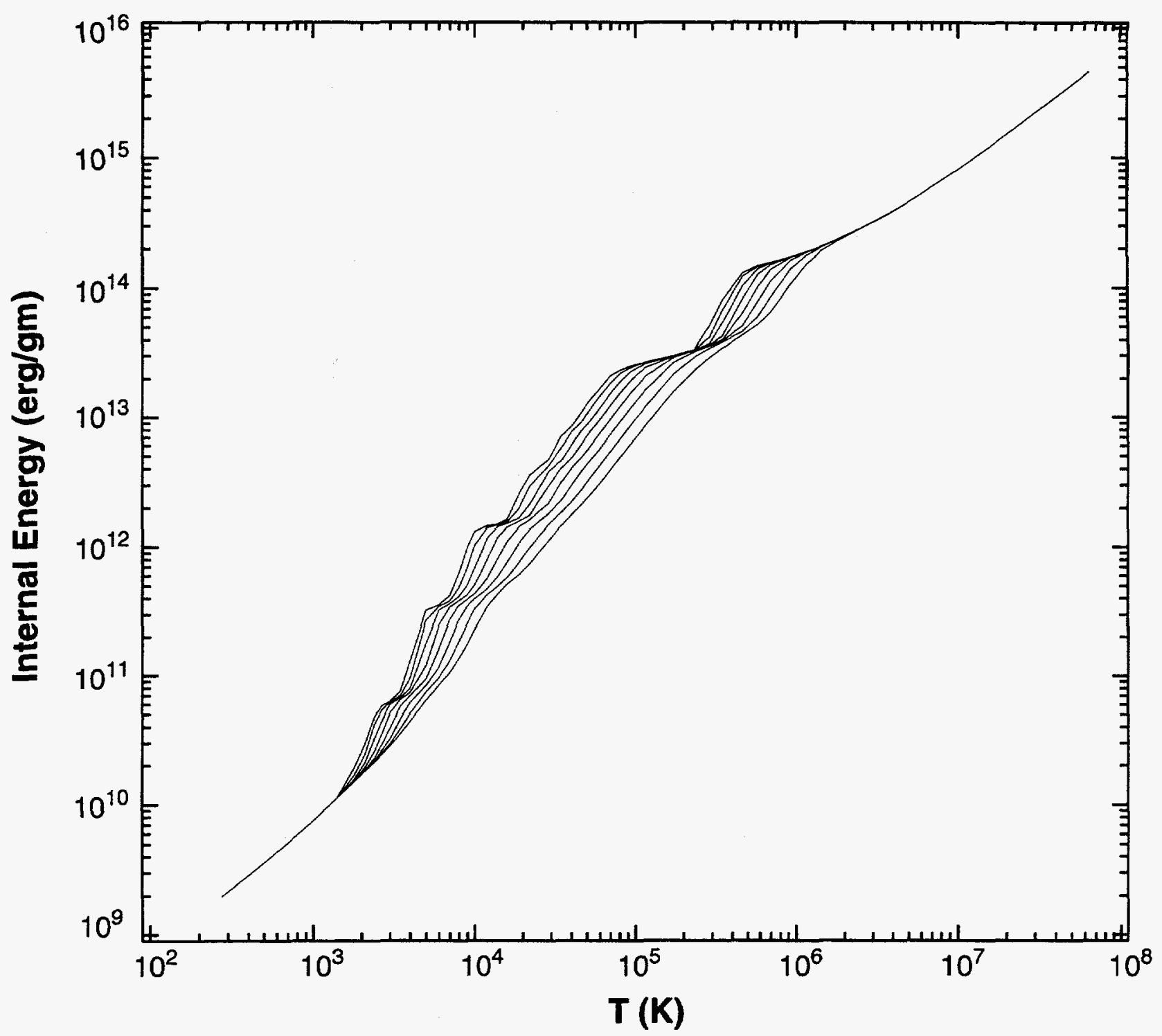

Figure 5. Plots of the internal energy vs temperature for eight atmospheric densities. The densities, left to right, are $10^{-2}, 10^{-3}, 10^{-4}, 10^{-5}, 10^{-6}, 10^{-7}, 10^{-8}$, and $10^{-9} \mathrm{gm} / \mathrm{cc}$. Ambient sea level density is $\approx 1.23 \times 10^{-3} \mathrm{gm} / \mathrm{cc}$. 


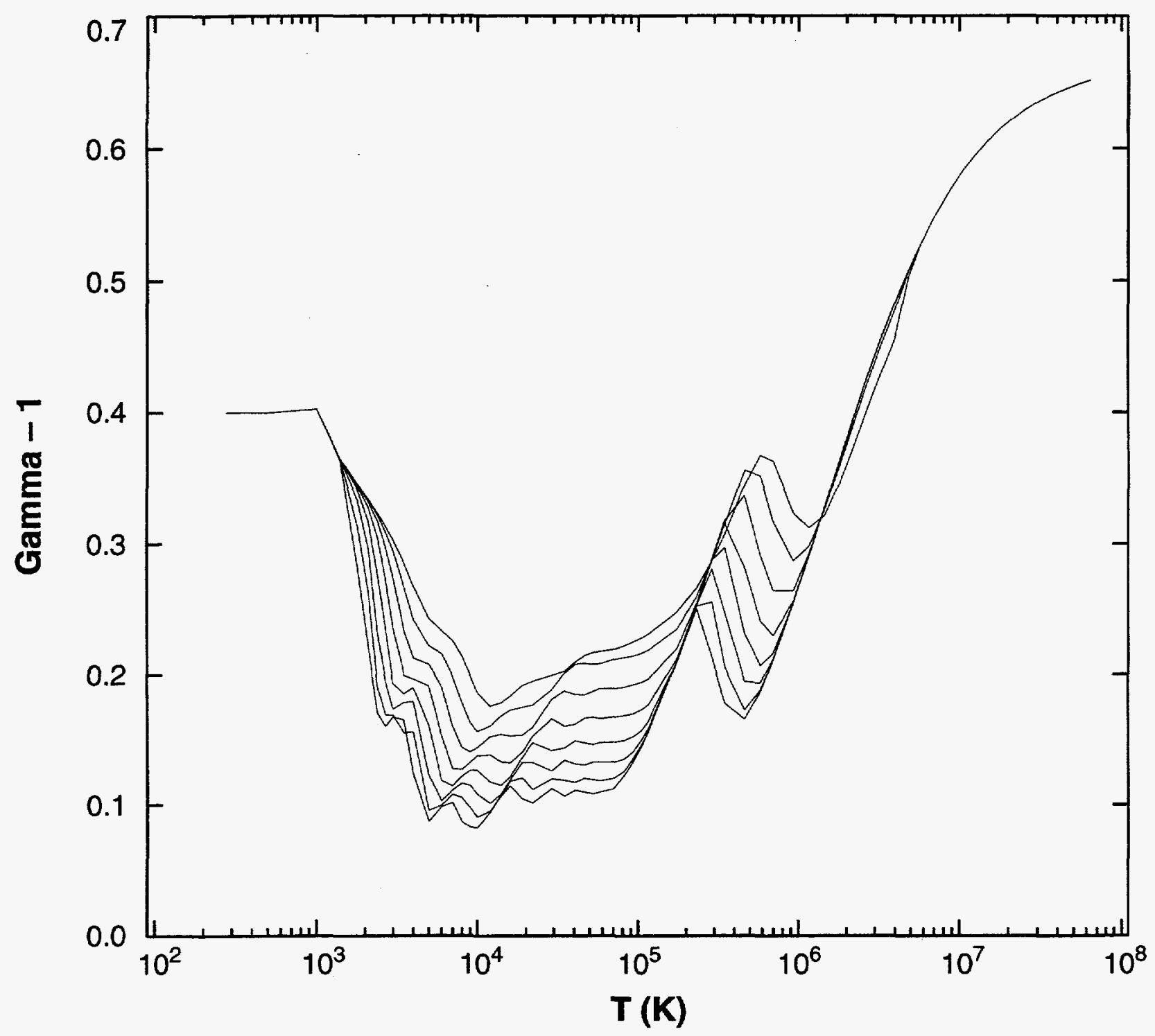

Figure 6. Plots of $(\gamma-1)$ vs temperature for eight atmospheric densities. The densities, top to bottom, are $10^{-2}, 10^{-3}, 10^{-4}, 10^{-5}, 10^{-6}, 10^{-7}, 10^{-8}, 10^{-9} \mathrm{gm} / \mathrm{cc}$. 
We next describe our one-dimensional analog of CAVEAT. CAVEAT is a timeexplicit, finite volume formulation of the Navier-Stokes equations. We ignore viscosity, heat conduction, and body force (gravity) terms. The differential equations, ignoring the radiative flux terms, are integrated over a finite volume element defining a discretized mesh. In onedimensional, spherical symmetry the volume elements are spherical shells. Let

1. $U C=$ the cell centered velocity

2. $r=$ the cell face radius

3. $E=$ the cell centered total energy

4. $e=$ the cell centered internal energy

5. $P=$ the cell centered pressure

6. $V=$ the cell volume $=4 \pi\left(r_{i+1}{ }^{3}-r_{i}^{3}\right) / 3$

7. $A=$ the cell face area $=4 \pi r^{2}$

8. $C M=$ the cell mass $=$ constant for each cell in a Lagrangian simulation

9. $D R=$ the cell width $=r_{i+1}-r_{i}$

The approximate Riemann solver-given the cell centered state variables, cell geometry, and an equation of state-computes

10. $U F=$ the cell face velocity

11. $P F=$ the cell face pressure

which are used to update, to time $t^{n+1}$, the state variables of cell $i$ by

$r_{i}^{n+1}=r_{i}+\Delta t U F_{i}$

$U C_{i}^{n+1}=U C_{i}-\Delta t\left(P F_{i+1}-P F_{i}\right) V_{i} /\left(C M_{i} D R_{i}\right)$

$E_{i}^{n+1}=E_{i}-\Delta t\left((P F \cdot A \cdot U F)_{i+1}-(P F \cdot A \cdot U F)_{i}\right)$

where everything on the right-hand side of the three above equations is evaluated at . $\Delta t$ is the computational time step equal to $t^{n+1}-t^{n}$. The new cell geometry can now be calculated and the mass density and the internal energy follows:

$\rho=C M / V, \quad e=E / C M-U C^{2} / 2$. 


\subsection{The SSW Patch}

The much greater complexity of the present RADFLO codes compared to the 1973 version is mainly associated with the "SSW patch"-the extra logical switches and computations necessary for deciding where the fireball front is, what its velocity is, how to correct for artificial viscous pressure or other numerical diffusive effects, and how to correct the computed radiation fluxes out of the fireball surface to correspond to the SSW values. It is beyond the scope of this report to describe the detailed reasons for the numerical oscillations in brightness and radiated power which the SSW patch is designed to avoid. They are discussed in detail in Zinn and Sutherland (1982). In brief outline they arise because of numerical smearing of the fireball front, which occurs in two different ways:

1. Relative to the dimensions of the numerical mesh cells (meters) the early fireball "front" is essentially a step function in temperature and density. The hot air behind the front is opaque to radiation at visible and IR wavelengths. In the computation, as the model front progresses through the mesh it is necessarily averaged at each instant across one or more cells, so that the cell that contains the front has an artificial temperature that increases with time from the initial ambient temperature up to the true fireball front temperature.

2. The hydrodynamics algorithm intentionally smears the shock front over several cells since it can't cope with a true discontinuity. In both the Richtmyer-von Neumann and Godunov hydro algorithms, the artificial smearing processes lead to a poorly determined fireball surface temperature and to oscillations in computed brightness.

To cope with the problem we had to devise ways to determine (1) where the actual fireball front is and (2) what its true temperature is, or alternatively, what its velocity is.

Both the artificial viscous pressure construct and the Godunov method have the important property that they produce the correct jump in density and entropy (or temperature) across the model shock front. They also produce a smooth transition in energy across the front, instead of a discontinuity. In doing so, they produce an artificially elevated energy and temperature just ahead of the shock- where is positive. The shock "front" lies within a cell where $\partial \rho / \partial t>0$ but where $\partial \rho / \partial t \leq 0$ in the cell just behind. In the cells just ahead of the shock (where $\partial \rho / \partial t>0$ ) there is artificial compressional heating, and the temperature is artificially elevated, while behind the shock (where $\partial \rho / \partial t \leq 0$ ) the temperature is correct. Thus, after each hydrodynamics step of the computation and before each radiation transport step, we must correct the cell internal energies $(E)$ for the 
artificial heating. To do this in the code, we maintain two separate cellwise energy arrays, the first being $E$, the energy used by the hydrodynamics subroutine, and the second being $e s h k$, the energy used by the radiation transport routine. $E$ is updated each time step, both by the hydrodynamics and radiation transport routines. eshk is identical to $E$ in the cells where $\partial \rho / \partial t \leq 0$, but in cells where $\partial \rho / \partial t>0$ it is updated only by the radiation transport. esh $k$ is a better approximation to the true internal energy than is $E$ (since it does not contain the artificial viscous heating). Still, as the necessary consequence of using a finite mesh, the transition in eshk at the fireball front is spread artificially over two or more cells. The temperatures used by the radiation transport routine are computed from eshk and the density rho-these temperatures are used in interpolating the absorption coefficients and computing Planck functions. For purposes of computing the fireball growth and profiles of temperature, density, velocity, etc., no other corrections are made for the numerical smearing of the front. However, for computing the fireball radiated power and visible and thermal brightnesses it is necessary to go a step further.

Because of the spreading of the fireball front temperature discontinuity over two or more cells, the brightness and power radiated by the front will still tend to oscillate as the front progresses through the mesh. The oscillation goes through one cycle for each mesh cell traversed. In HYCHEM (or RADFLO $\mathrm{Z}_{\mathrm{Z}}$ ) to deal with this problem and to compute the "correct" brightnesses for wavelengths longer than $180 \mathrm{~nm}$, we proceed as follows: as the computation runs we attempt to determine at each numerical time step (1) where the true fireball front is and (2) what its velocity is. From this velocity and a stored tabulation of effective radiating temperature vs front velocity (as derived from the separate SSW computations) we interpolate to obtain the effective radiating temperature of the front. This radiating temperature includes the absorption by the fine-scale radiative precursor, which is resolved in the SSW computations but is much too thin to be resolved in the coarse zones used by RADFLO. This effective radiating temperature is used to compute the effective blackbody flux out of the fireball surface for each of the RADFLO quantum energy groups (but limited to wavelengths longer than $180 \mathrm{~nm}$ ). The radiation transport routine is run in the usual way, except that we substitute the SSW effective blackbody flux at the outer vertex of the cell that contains the front. This flux may then be modified by the normal transport algorithm to account for absorption by preheated air outside the fireball front (i.e. the x-ray veil, and/or by "smog" in the case of HYCHEM computations). As a further fussy detail, we also modify the computed absorption that arises from the cell containing the front 
because that cell has a distorted temperature arising from the abovementioned front-spreading.

Our previously published SSW computations were only for shocks and thermal waves in sea level-density air. However, in order to use the SSW patch algorithm in HYCHEM computations for altitudes above sea level, we have recently run additional SSW computations for a range of air densities. The results of these computations are outlined in Appendix A. Qualitatively they show that as the air density decreases the shock velocity for maximum shock brightness decreases, and the magnitude of the maximum brightness also decreases. On the other hand, in the high-velocity thermal-wave regime the wavefront brightness for a given velocity increases with decreasing air density. These results are incorporated in tables within HYCHEM, and the SSW patch algorithm has been extended to include the background air density as a variable in addition to the wavefront velocity.

\subsubsection{The RADFLO Patch}

The RADFLO $_{S}$ code also contains a fireball brightness patch algorithm which is designed to give an approximate fit to the SSW results from Zinn and Sutherland (1981), relating to the fireball front radiative precursor attenuation for sea level air-density cases, but at the same time provide a guess as to the amount of precursor attenuation that would be expected in high-altitude cases. That algorithm of course predates our recent extended set of SSW computations which include other than sea level air densities.

\subsection{Time Steps}

The basic numerical time step $d t$ is dictated by requirements of numerical stability, as described by the familiar Courant conditions for the hydrodynamics and analogous conditions for the radiation transport. Generally it takes many time steps for the fireball front to progress from one cell to the next. We also refer to a "macro" time step-namely the time required for the front to cross a cell. $d t$ refers to the "micro" time step. At each micro time step we determine the index lzero of the cell that contains the fireball front. The algorithm for defining lzero is fairly complex, but basically it is the larger of two indices, irmx and itmx, where irmx labels the outermost cell with a density that exceeds a certain threshold indicating an "air shock," and itmx labels the cell that contains the largest temperature gradient. As the model run progresses, the times and radial positions at which lzero advances from each cell to the next are stored in a pair of arrays, and the fireball front velocity is obtained as the finite difference derivative - one value for each macro time 
step. These velocities are used to interpolate in the SSW-generated tables of effective radiating temperature vs velocity to obtain the effective fireball temperature for the brightness and radiated power computation. The radiated flux patch is applied at the cell vertex just outside of lzero.

\subsection{Chemistry}

In HYCHEM, if the input parameter ichem is set to 1 , the run will include chemistry - a set of chemical-reaction kinetics equations is integrated for a predetermined set of chemical species (currently 45) for a predetermined set of time steps. The results include profiles of the concentrations of these species at the predetermined output times. The chemistry outside the fireball is driven largely by neutron- and gamma ray-induced ionization of the air and ensuing chemical reactions. Therefore the code necessarily includes algorithms for computing the prompt neutron and gamma ray emission and transport and the bombdebris radioactive-decay gammas. An important effect of the chemistry is to produce optically-absorbing chemical species outside the fireball, which tend to absorb radiation emitted by the fireball and thereby reduce the radiated power. These species include $\mathrm{NO}_{2}, \mathrm{O}_{3}$, and $\mathrm{HNO}_{2}$, and in fireball vernacular they are called "smog."

The chemistry computations are done at prescribed intervals of time which are much larger than the micro time step used in the main rad-hydro computation. After the chemical concentrations have been computed, we can compute the changes in the radiative absorption coefficients resulting from the deviations from chemical equilibrium. The equilibrium chemical composition is determined by a separate subroutine that computes the composition that gives the minimum value of the Gibbs free energy. Then at each mesh cell at each micro time step, the difference between each concentration and the equilibrium value is used together with a set of tables of absorption cross sections to correct the normal equilibrium radiative-absorption coefficients. In this way the radiation transport is modified by the chemistry, and the effects of nuclear smog outside the fireball are computed. The smog absorption can have a very large effect on the radiated power from a large-yield fireball at early times.

The chemical reaction rate equations include photochemical reactions associated with radiation emitted by the fireball. The reaction rates are recomputed at each chemistry time step to take account of temperature changes. The chemistry and gamma ray+neutron transport algorithms are described in some detail in Zinn et al. (1982) along with the chemical species set and the reactions and rate coefficients. The algorithm for the neutron transport in 
the present HYCHEM code is quite crude and probably constitutes the most serious source of error in the chemistry and smog computations. At one time we had hoped to add a detailed neutron transport subroutine based on the standard code MCNP.

\subsection{The RADFLO Smog Model}

The RADFLO $\mathrm{S}_{\mathrm{S}}$ code also includes an algorithm for smog absorption, although no chemistry computations are done. This algorithm (Sappenfield 1976) is based on the analysis of data by C. K. Mitchell from the Navajo and Flathead chord experiments, in which it was assumed that the smog optical-absorption coefficient was directly proportional to the timeintegrated gamma ray and neutron energy deposition, with no other time dependence (Mitchell 1979). The gamma ray- and neutron-energy deposition is computed with an algorithm from the DNA ROSCOE code (Knapp et al. 1974).

\subsection{Debris Opacities}

Since the tabulated opacity data are for air, the radiation transport in the early bomb vapor and later bomb-debris-air mixture are treated only approximately. During the early period of $x$-ray emission by the bomb, we multiply the absorption coefficients within the debris by an arbitrary factor of ten to assure that the bomb emits as a blackbody. For later times we add extra terms to the opacity in the debris region to account for the expected higher free electron concentrations (due to the lower ionization potentials of debris materials) and the expected large number of allowed low-energy bound-bound transitions. For the bound-bound transitions we assume an average absorption cross section of $1.3 \times 10^{-18} \mathrm{~cm}^{2}$ over the range $h v=1$ to $4 \mathrm{eV}$, which assumes that each debris atom has one allowed transition in this range with an oscillator strength of $10^{7} \mathrm{~s}^{-1}$. The extra terms for the enhanced free electron concentration include (1) enhanced free-free absorption in the fields of neutral atoms and (2) enhanced bound-free absorption due to negative $\mathrm{O}^{-}$ions. We also estimate the size of the debris region, since the debris expansion is assuredly Taylor-unstable, using an algorithm that says that the debris tends to mix with air up to 20 times its own mass.

\subsection{Accessory Subroutines}

In addition to the main computations of fireball growth and chemistry, the code includes accessory routines for things such as (1) computing profiles of fireball brightness at visible wavelengths, (2) storing tables of fireball radii, velocities, and radiated power vs time for later plotting, and (3) input and output. The code also includes subroutines for adjusting the mesh as the problem runs-i.e., (1) combining cells behind the front to improve 
running speed, and (2) adding cells at the outside of the mesh when the shock wave gets close to the outer boundary. These routines are necessary to enhance running speed and spatial/temporal resolution.

\subsection{INITIAL CONDITIONS}

The yield of a given device is nominally partitioned in terms of an x-ray yield, a kinetic energy (of the debris) yield, and an internal energy (of the debris) yield. ${ }^{1}$ In addition a gamma ray yield and a neutron yield may be specified.

In $\mathrm{RADFLO}_{z}$, the algorithm for initialization of the explosion is based on a very simple model for a generic nuclear bomb and has been described previously in Zinn (1973). We specify the device yield $(\mathrm{Y})$, the mass $(\mathrm{M})$, the average density $\left(\rho_{s}\right)$, and a time $\left(\tau_{x}\right)$ representing the time duration of the nuclear reaction. We further assume that the device is spherical, and that the energy $(\mathrm{Y})$ is deposited uniformly within the device mass so that the density and temperature remain uniform. The $\mathrm{x}$-rays from the explosion are emitted at a constant rate over the time $\left(\tau_{x}\right)$ with a Planckian distribution characterized by a fixed temperature $T_{x}$ (which is to be computed). In addition we assume that the energy remaining after $\mathrm{x}$-ray emission is apportioned equally between internal and kinetic. To compute $T_{x}$ we also need to know the effective specific heat for raising the bomb mass to temperature $T_{x}$, and the radiating surface area, which is obtained from the given mass and given density. Given these assumptions it is then straightforward to calculate the radiating temperature $T_{x}$ and the partition of the energy $Y$ between (1) radiated, (2) internal, and (3) kinetic. The equations are given in Zinn (1973).

\subsection{X-ray Deposition}

A special subroutine is used for computing the distribution of energy deposition in the air due to the bomb $\mathrm{x}$-rays. In the case of a nuclear explosion at sea level, the electron collisions are fast enough to assure that the air stays in thermodynamic equilibrium as the $\mathrm{x}$ rays are deposited. The air-absorption coefficients change as the air heats up, but they can be computed in the usual way from the equilibrium air-opacity tabulation. The evolution of

\footnotetext{
1 The word debris is synonymous with device or bomb in this report. In $\mathrm{RADFLO}_{\mathrm{s}}$, the first jdeb number of zones are debris zones. The user must supply an internal energy and kinetic energy to each of them. The default equation of state for the debris is the same as the air equation of state.
} 
the deposition process must be done stepwise to follow the dynamically changing absorption coefficients. At each step the x-ray energy that is transported and deposited in the air is, at the same time, removed from the bomb. The radiation transport is computed in the usual way by the algorithms already described. The stepwise computation is continued until the previously determined $\mathrm{x}$-ray yield has been transferred from the bomb to the air. In the case of a high-altitude explosion (about $40 \mathrm{~km}$ or higher), electron collisions are not sufficiently fast to maintain thermodynamic equilibrium on the time scale of the bomb xray emission. For such cases it is a better approximation to assume that the air-absorption coefficients are those of cold air-except where the energy deposition density is so large as to exceed the energy of four $\mathrm{x}$-ray photons per air atom, in which case the absorption coefficient drops to a very small value (the bremsstrahlen limit). The four-photon limit is based on the expectation that each $\mathrm{x}$-ray photon absorbed (in the $\mathrm{K}$ shell) results in ejection of two electrons-a photolectron and an Auger electron, and the $\mathrm{K}$ shell is immediately refilled from outer shells until there are no more outer shell electons. In the case of oxygen, which has eight electrons per atom, this permits four photons to be absorbed.

\subsection{HYCHEM Input Parameters}

In the input to the HYCHEM code the bomb, or device, is described by 12 parameters as listed in Table I. The most important of these are (1) the yield (yield), (2) the bomb mass (bmbms), (3) the average bomb density (rhol), (4) the x-ray radiating time (taux), and (5) the fraction of the non- $x$-ray yield (or non-radiated) yield that ends up as kinetic energy (cekin/2). In addition there are three prompt gamma ray parameters, namely (6) the fraction of the device yield emitted as prompt gammas (fgam), (7) the approximate gamma ray straggle time (dtgam), and (8) the average gamma ray mass absorption coefficient (akappa). Also, there are three prompt neutron parameters, namely (9) the neutron yield fraction (fneut), (10) the average neutron velocity (vneut), and (11) the average neutron mean free path (alam). Finally, there is (12) the fission yield fraction (ffiss), which is used for computing the delayed fission-product decay, beta- and gamma-emission rates. If chemistry is not included in the computation, only the first five of these parameters are relevant. In the input subroutine all the bomb parameters except yield are provided with default values, in case they are not otherwise specified. The default values are also listed in Table I.

The computational mesh is set up with an automatic algorithm which is controlled by four input parameters, namely: (1) the number of cells containing bomb material (ndc), 
Table I. HYCHEM Input Variables.

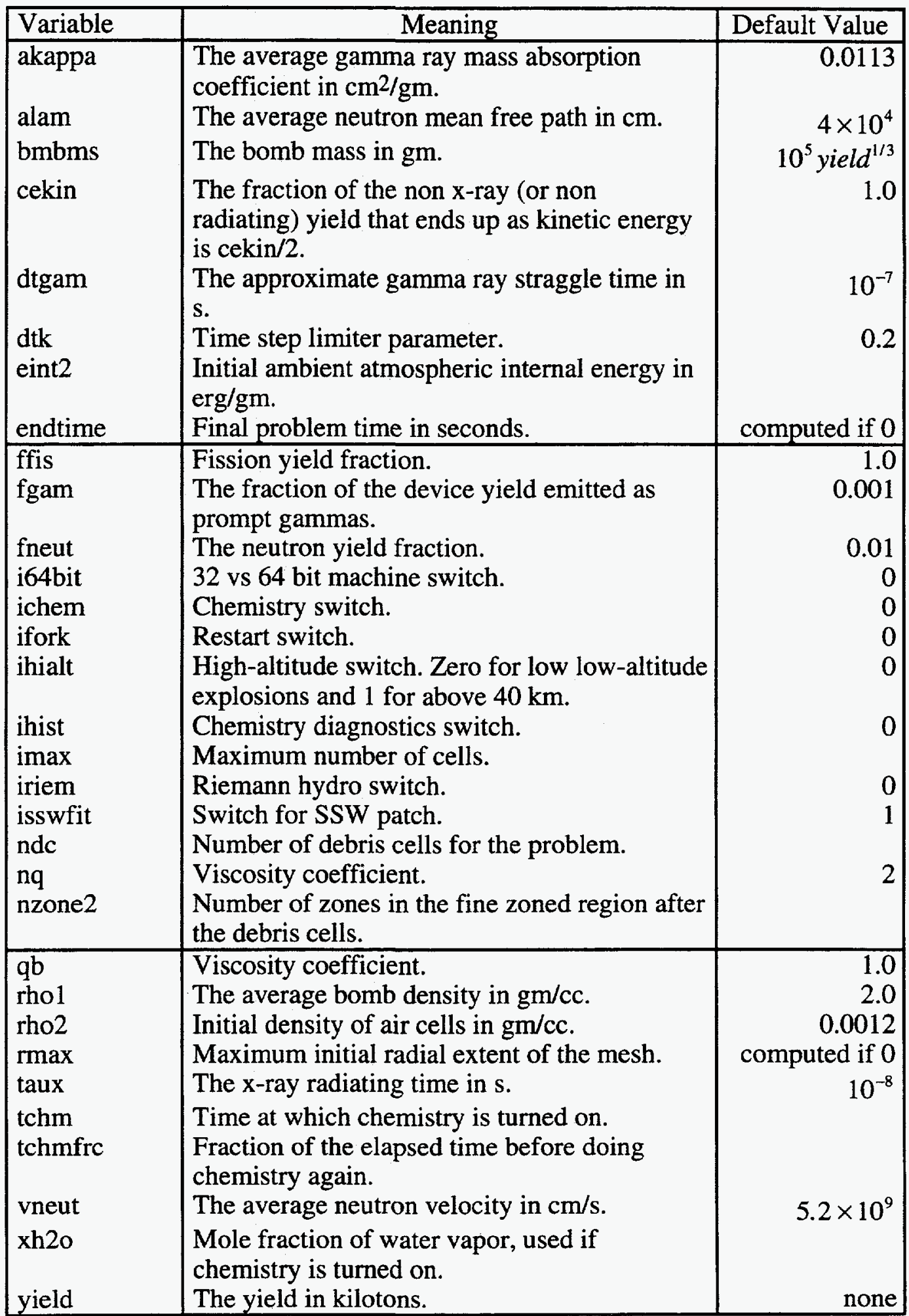


(2) the number of cells between the bomb surface and the radius at which the average fireball temperature is about $30 \mathrm{eV}$ (nzone2), (3) the total number of cells out to the outermost mesh boundary (imax), and (4) the radius of the outermost boundary ( $\max$ ). If $r$ max is not otherwise specified it is set with a default expression $r \max =780 \times(\text { yield } / r h o 2)^{1 / 3} \mathrm{~cm}^{2} . r$ ho 2 is the background air density $(\mathrm{g} / \mathrm{cc})$, which must also be specified. The $30 \mathrm{eV}$ radius is computed

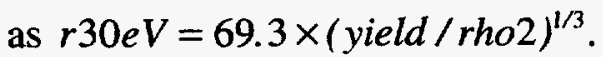

The zoning algorithm computes the initial cell vertex radii so that (1) the $n d c$ bomb debris cells contain equal masses, whose sum is $b m b m s ;(2)$ for the next nzone 2 cells, each cell mass is a constant multiple dmfctr 2 times the mass in the previous cell; (3) likewise, for the remaining nzone 3 cells, each cell mass is a constant factor $d m f c t r 3$ times the mass of the previous cell.

\subsection{RADFLO Input Parameters}

RADFLO $_{S}$ assumes the user of the code is fairly knowledgeable about the device being modelled and thus has a large number of input parameters for fine tuning the simulation. We list them in Tables II and III and will describe them in detail in the User's Manual report (Horak 1983).

\subsection{SOME NUMERICAL RESULTS}

In order to test the current code and to benchmark a reference set of computed results,

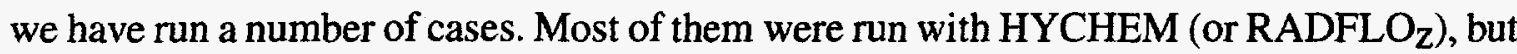
a few were run with RADFLO . The cases include yields ranging from 0.01 kilotons to 1 Megaton, and altitudes ranging from sea level up to $50 \mathrm{~km}$ at a few different device masses. In most cases we have not included the nonequilibrium chemistry (standard RADFLO mode), but in a few instances we ran identical cases both with and without the chemistry.

Since this is an unclassified report which describes the computer code and general aspects of fireball physics, we will avoid any quantitative references to nuclear test data. The computations are all based on our hypothetical model of a generic bomb, as described above. In most cases we have used the default values of bomb mass, etc. However, in a few cases we investigated effects of varied bomb masses. In RADFLO $\mathrm{S}_{\mathrm{S}}$ cases we attempted to match the input parameters as closely as possible with an equivalent run done with $\mathrm{RADFLO}_{Z}$, so that the results would be directly comparable.

In this section we describe results relating to optical observables-especially optical power vs time (bhangmeter curves), and fireball radius vs time. 
Table II. RADFLO Input Variables. Most of the variables are defined as they were in Sappenfield (1984).

\begin{tabular}{|c|c|c|}
\hline Variable & Meaning & Default Value \\
\hline cno2 & $\begin{array}{l}\text { Factor for determining } \mathrm{NO}_{2} \text { concentrations in } \\
\text { smog routines. }\end{array}$ & $\begin{array}{l}\text { Depends on } \\
\text { fgfact/fnfact }\end{array}$ \\
\hline dfrac & $\begin{array}{l}\text { The fraction of the shock radius at which the } \\
\text { outer boundary of the debris is assumed to be, } \\
\text { prior to the time at which the debris boundary is } \\
\text { frozen in Langrangian space. }\end{array}$ & 1.0 \\
\hline & $\begin{array}{l}\text { Gridding array. } \\
\text { Initial hydro time step. }\end{array}$ & $10^{-6} \mathrm{~s}$ \\
\hline $\begin{array}{l}\text { ekin0 } \\
\text { eofx(jdeb) } \\
\text { est }\end{array}$ & $\begin{array}{l}\text { Initial kinetic energy in kilotons. } \\
\text { Initial bomb internal energies. } \\
\text { Ergs/gm required to strip an air atom at } \\
\text { equilibrium. }\end{array}$ & none \\
\hline fact & Total gamm ray and neutron yield (ergs). & 0.0 \\
\hline feng1 & $\begin{array}{l}\text { The maximum fractional internal energy change } \\
\text { per time step due to radiation flow that is } \\
\text { allowed initially. }\end{array}$ & \\
\hline feng2 & The value to which $f c n g l$ is changed at $t f c n g l$. & $0.5 * f c n g 1$ \\
\hline ffrc & Fission fraction. & 0.5 \\
\hline $\begin{array}{l}\text { fgfact } \\
\text { fh }\end{array}$ & $\begin{array}{l}\text { Fractional yield in gamma rays. } \\
\text { Fraction of total energy in the spectrum with } \\
\text { temnerature th }\end{array}$ & 0.0 \\
\hline fnfact & Fractional yield in neutrons. & 0.0 \\
\hline 1 & Flag for new start or restart. & none \\
\hline $\mathrm{idb}$ & $\begin{array}{l}\text { Flag indicating treatment of debris contribution } \\
\text { to opacity. }\end{array}$ & none \\
\hline idet & Non LTE calculation of $\mathrm{NO}_{2}$ switch. & none \\
\hline id 3 & tbloop option swtich. & \\
\hline $\begin{array}{l}\text { ieinc } \\
\text { ihop }\end{array}$ & $\begin{array}{l}\text { Flag for } \mathrm{x} \text {-ray deposition. } \\
\text { Flag to bypass all radiation transport }\end{array}$ & 0 \\
\hline & $\begin{array}{l}\text { calculations. A value of } 1 \text { implies a hydro only } \\
\text { simulation. }\end{array}$ & \\
\hline $\begin{array}{l}\mathrm{ijz} \\
\mathrm{im}\end{array}$ & Number of values of dr(i) to read in & \\
\hline & Flag for plotting abosorption coefficients. & none \\
\hline $\begin{array}{l}\text { iprint } \\
\text { irez }\end{array}$ & $\begin{array}{l}\text { Flag for simulations prints. } \\
\text { Flag controlling rezone. A value of zero } \\
\text { prohibits rezoning. }\end{array}$ & \\
\hline $\begin{array}{l}\text { irite } \\
\text { istop }\end{array}$ & $\begin{array}{l}\text { Controls frequency of binary data dumps. } \\
\text { Flag to stop simulation after } \mathrm{x} \text {-ray deposition. }\end{array}$ & 0 \\
\hline itmax & $\begin{array}{l}\text { Number of entries in temperature table, } \\
\text { currently hardwired to } 63 \text {. }\end{array}$ & 63 \\
\hline
\end{tabular}


Table III. More RADFLO Input Variables.

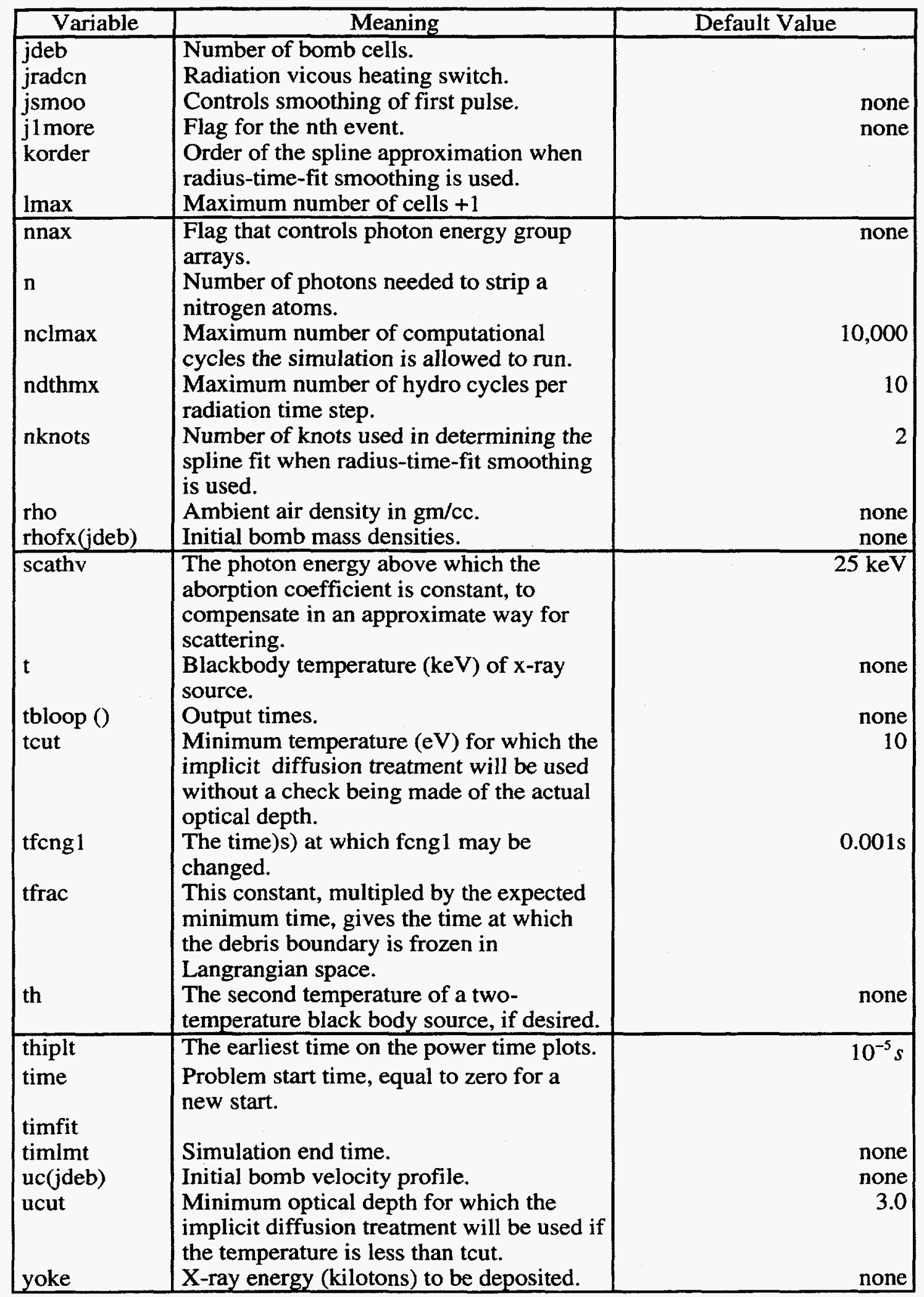


A nuclear explosion in air at near-sea level densities produces a distinctive fireball optical power output vs time signature consisting of two pulses with a deep minimum in between. ${ }^{2}$ (The same statement applies for infrared and near-UV wavelengths.) For explosions at a given altitude the time of the power minimum is uniquely related to the bomb yield, and the same is true for the second maximum. Thus by measuring the minimum time or the second maximum time one can measure the yield. For altitudes above sea level the depth of the minimum decreases with increasing altitude until, at about $40 \mathrm{~km}$ (but depending on yield), the minimum disappears altogether.

The RADFLO and HYCHEM codes compute, among other things, the fireball radiated power (in several wavelength bands) as a function of time, and also the fireball visual radius vs time. Table IV summarizes the sea level air density cases that we have run-with and without the nonequilibrium chemistry. The quantities tabulated include the input device yield and mass and the timings of seven different phenomenological features, most of which are evident in the computed optical power vs time curves. Table V summarizes the high-altitude cases we have run. In a few cases there are entries both from a HYCHEM run and from an equivalent RADFLO run. $_{S}$

Figure 7 is a log-log plot of the computed first maximum, minimum, and second max times vs the bomb yield for the sea level cases. ${ }^{3}$ For the HYCHEM runs, the minimum and second max time points are fitted very well by a pair of log-log straight lines, except for very small yields below $1 \mathrm{kt}$, where the $t_{\min }$ points fall below the line. The two straight lines are given by the equations

$t_{\min }(S i)=0.00430 Y^{0.414}$ seconds

and

$t_{2 \max }(S i)=0.0450 Y^{0.441}$ seconds.

\footnotetext{
${ }^{2}$ Note that the optical power (in Watts) is approximately equal to the product of the fireball surface area times the optical brightness (in Watts $/ \mathrm{cm}^{2}$ steradian) times $\pi$. So the power and the brightness (or luminosity) are not to be confused.

${ }^{3}$ Here the spectral distribution is chosen as that of an unfiltered silicon photodetector.
} 
Table IV. Summary of computed optical power results, for silicon spectral response, for explosions at sea level altitude. This table was constructed from RADFLO simulations using the original hydro algorithm. Twelve debris zones were used for each simulation.

\begin{tabular}{|c|c|c|c|c|c|c|c|c|}
\hline \begin{tabular}{|l|}
$\begin{array}{l}\text { Yield } \\
(\mathrm{kt})\end{array}$ \\
\end{tabular} & $\begin{array}{l}\text { Mass } \\
\text { (g) }\end{array}$ & $\begin{array}{l}t_{\text {Imax }} \\
\text { (s) }\end{array}$ & $\begin{array}{l}t_{\min } \\
(\mathrm{s})\end{array}$ & $\begin{array}{l}t_{2 \max } \\
(\mathrm{s})\end{array}$ & $\begin{array}{l}t_{\text {veil }} \\
(\mathrm{s}) \\
\end{array}$ & $\begin{array}{l}t_{50 \mathrm{~km} / \mathrm{s}} \\
(\mathrm{s})\end{array}$ & $\begin{array}{l}t_{\text {dbshkc }} \\
\text { (s) }\end{array}$ & $\begin{array}{l}t_{0.8 \mathrm{eVbp}} \\
(\mathrm{s})\end{array}$ \\
\hline \multicolumn{9}{|c|}{ 1. Runs at sea level air density without chemistry. } \\
\hline 0.02 & $2.7 \mathrm{E}+4$ & 3.E-5 & $5.3 \mathrm{E}-4$ & $8.5 \mathrm{E}-3$ & - & 2.E-5 & - & - \\
\hline 0.10 & $4.6 \mathrm{E}+4$ & 4.E-5 & $1.0 \mathrm{E}-3$ & $1.6 \mathrm{E}-2$ & - & $2.5 \mathrm{E}-5$ & - & - \\
\hline 0.20 & $5.8 \mathrm{E}+4$ & 5.E-5 & $1.4 \mathrm{E}-3$ & $2.2 \mathrm{E}-2$ & - & $3.2 \mathrm{E}-5$ & 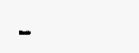 & $1.1 \mathrm{E}-3$ \\
\hline 0.30 & $6.7 E+4$ & 5.E-5 & $2.5 \mathrm{E}-3$ & $2.6 \mathrm{E}-2$ & - & $1.9 \mathrm{E}-5$ & $2.3 \mathrm{E}-5$ & $1.1 \mathrm{E}-3$ \\
\hline 1.0 & $1.0 \mathrm{E}+5$ & $5.5 \mathrm{E}-5$ & $4.5 \mathrm{E}-3$ & $4.5 \mathrm{E}-2$ & - & $2.3 \mathrm{E}-5$ & $4.4 \mathrm{E}-5$ & $1.1 \mathrm{E}-3$ \\
\hline 2.0 & $1.3 \mathrm{E}+5$ & 7.E-5 & $5.5 \mathrm{E}-3$ & $6.2 \mathrm{E}-2$ & - & $2.7 \mathrm{E}-5$ & $7.0 \mathrm{E}-5$ & $1.2 \mathrm{E}-3$ \\
\hline & $2.5 \mathrm{E}+5$ & 8.E-5 & $"$ & & 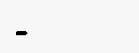 & $3.3 \mathrm{E}-5$ & & $1.4 \mathrm{E}-3$ \\
\hline " & $5.0 \mathrm{E}+5$ & $1.0 \mathrm{E}-4$ & $"$ & $"$ & 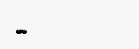 & 7.E-5 & - & $2.1 \mathrm{E}-3$ \\
\hline " & $1.0 \mathrm{E}+6$ & $1.1 \mathrm{E}-4$ & $"$ & $"$ & - & - & - & - \\
\hline " & $2.0 \mathrm{E}+6$ & 1.2E-4 & $"$ & $"$ & - & - & - & - \\
\hline " & $4.0 \mathrm{E}+6$ & $1.8 \mathrm{E}-4$ & $"$ & $"$ & - & - & - & 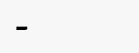 \\
\hline " & $8.0 \mathrm{E}+6$ & $2.8 \mathrm{E}-4$ & " & $"$ & - & - & - & 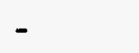 \\
\hline 20.0 & $2.7 \mathrm{E}+5$ & $2.5 \mathrm{E}-4$ & $1.4 \mathrm{E}-2$ & $1.7 \mathrm{E}-1$ & - & $4.5 \mathrm{E}-5$ & 2.E-4 & 2.E-3 \\
\hline " & $5.0 \mathrm{E}+5$ & 2.0E-4 & 1.4E-2 & $1.7 \mathrm{E}-1$ & & $4.5 \mathrm{E}-5$ & 2.E-4 & 2.E-3 \\
\hline 100.0 & $4.6 \mathrm{E}+5$ & $6.0 \mathrm{E}-4$ & $2.9 \mathrm{E}-2$ & $3.5 \mathrm{E}-1$ & 9.E-6 & $5.5 \mathrm{E}-5$ & 6.E-4 & 9.E-3 \\
\hline 1000.0 & $\begin{array}{l}1.0 \mathrm{E}+6 \\
2.0 \mathrm{E}+6\end{array}$ & $1.7 \mathrm{E}-3$ & $7.5 \mathrm{E}-2$ & $9.5 \mathrm{E}-1$ & $\begin{array}{l}3 . \mathrm{E}-4 \\
1.5 \mathrm{E}-4\end{array}$ & $9.5 \mathrm{E}-5$ & $1.5 \mathrm{E}-3$ & $2.0 \mathrm{E}-2$ \\
\hline " & $4.0 \mathrm{E}+6$ & $1.1 \mathrm{E}-3$ & $7.5 \mathrm{E}-2$ & $9.5 \mathrm{E}-1$ & 3.E-5 & $1.2 \mathrm{E}-4$ & 1.E-3 & 2.E-2 \\
\hline 5000.0 & $5.0 \mathrm{E}+6$ & $3.4 \mathrm{E}-3$ & $1.5 \mathrm{E}-1$ & & 6.E-4 & $1.5 \mathrm{E}-4$ & 2.E-3 & 3.E-2 \\
\hline \multicolumn{9}{|c|}{ 2. Runs at sea level air density with chemistry. } \\
\hline & & $2.5 \mathrm{E}-4$ & $1.4 \mathrm{E}-2$ & $1.7 \mathrm{E}-1$ & - & 4.E-5 & $2.5 \mathrm{E}-4$ & $1.8 \mathrm{E}-3$ \\
\hline 100.0 & $4.6 \mathrm{E}+5$ & $5.5 \mathrm{E}-4$ & $2.9 \mathrm{E}-2$ & $3.5 \mathrm{E}-1$ & 9.E-6 & $5.5 \mathrm{E}-5$ & 6.E-4 & 9.E-3 \\
\hline 1000.0 & $1.0 \mathrm{E}+6$ & $1.5 \mathrm{E}-3$ & $7.5 \mathrm{E}-2$ & $1.0 \mathrm{E}+0$ & $3.5 \mathrm{E}-4$ & $9.5 \mathrm{E}-5$ & $1.5 \mathrm{E}-3$ & $1.8 \mathrm{E}-2$ \\
\hline
\end{tabular}

Table V. Summary of computed optical power results, for silicon spectral response, for high-altitude explosions.

\begin{tabular}{|lll|llll|}
\hline $\begin{array}{l}\text { Yield } \\
(\mathrm{kt})\end{array}$ & $\begin{array}{l}\text { Mass } \\
(\mathrm{g})\end{array}$ & $\begin{array}{l}\text { altitude } \\
(\mathrm{km})\end{array}$ & $\begin{array}{l}t_{\max } \\
(\mathrm{s})\end{array}$ & $\begin{array}{l}t_{\min } \\
(\mathrm{s})\end{array}$ & $\begin{array}{l}t_{2 \max } \\
(\mathrm{s})\end{array}$ & $\begin{array}{l}t_{\text {dbshkc }} \\
(\mathrm{s})\end{array}$ \\
\hline 15. & $5.0 \mathrm{E}+5$ & 21. & - & $6.3 \mathrm{E}-3$ & $5.0 \mathrm{E}-2$ & $7 . \mathrm{E}-4$ \\
100. & $4.6 \mathrm{E}+5$ & 50. & - & - & $5 . \mathrm{E}-4$ & - \\
\hline
\end{tabular}




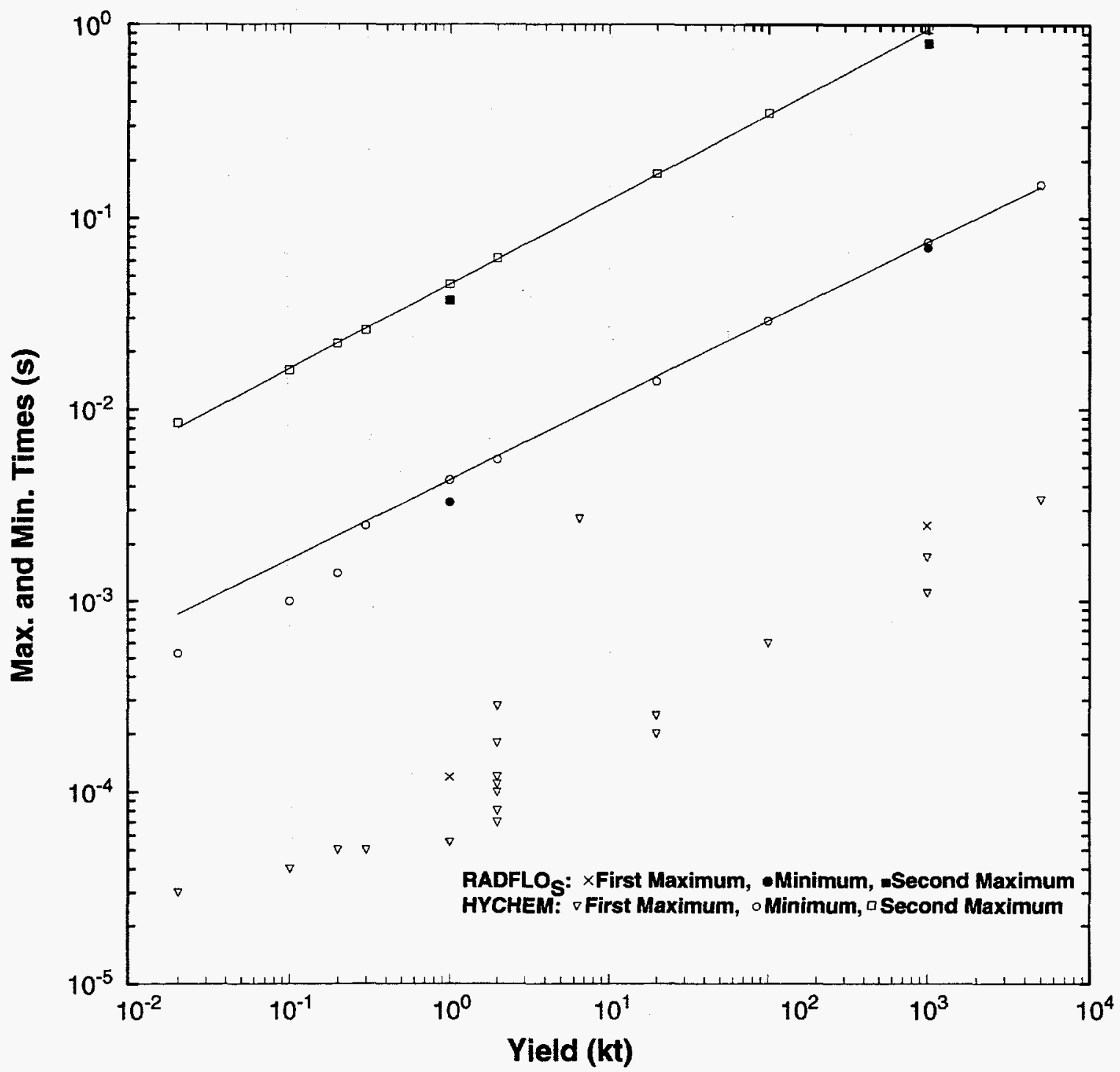

Figure 7. Plots of the times of first maximum, minimum, and second maximum (in seconds) as functions of the yield (in kilotons), as computed for the spectral response function of an unfiltered silicon photodetector for the cases in Table IV. The straight lines are scaling expressions fitted to the HYCHEM points (see text). 
The computed minimum and second max times are not affected by nonequilibrium chemistry inclusion or by the bomb mass - at least within the range of bomb mass values that we have tried.

For the cases from RADFLOS both the $t_{\text {min }}$ and $t_{2 \max }$ points fall slightly below the HYCHEM points, consistent with the observation, noted previously, that the RADFLO opacities in the relevant temperature and quantum energy range are slightly smaller than the HYCHEM opacities. Presumably if more RADFLOS $\mathrm{S}_{\mathrm{S}}$ cases were run the $t_{\min }$ and $t_{2 \max }$ points would also fall along a pair of straight lines, lying slightly below the two scaling law lines in Fig. 7.

Although the minimum and second maximum times scale neatly with the yield and are independent of the bomb mass, that is not true for the first maximum, as can be seen in Fig. 7 and Table IV. And, for the range bomb masses that we have run, seems to increase with increasing bomb mass for a yield of $2 \mathrm{kt}$ but decreases with increasing bomb mass for yields of $20 \mathrm{kt}$ and $1 \mathrm{Mt}$.

Most of the energy radiated by a sea level fireball comes out in the second pulse, and the total is roughly $30 \%$ of the bomb yield. Since the second maximum time varies as $Y^{0.4}$, it follows that the radiant power at second maximum varies roughly as $\mathrm{Y}^{0.6}$. For large yields the computed power is somewhat reduced when chemistry is included.

As noted earlier, for a given yield the time to first maximum depends on the device mass and other design details. Moreover, in these idealized computations, which assume a spherically symmetric bomb, the first maximum (in the case of the HYCHEM runs) often shows distinct fine-structure features:

1. On the rise before first maximum, there is usually a shoulder or a subsidiary maximum that occurs when the shock velocity, which is falling rapidly, passes through $50 \mathrm{~km} /$ $\mathrm{s}\left(t_{50 \mathrm{~km} / \mathrm{s}}\right.$ in Table IV). The brightness of a shock is a maximum at that velocity (in sea level-density air) (see Zinn and Anderson 1973 and Appendix A). After this point the power usually flattens out or decreases slightly.

2. Soon thereafter the inner debris-driven shock wave catches up with the outer fireball shock, causing its velocity and brightness to increase (in Table IV). In many cases this time is also the time of maximum power-i.e., the main first maximum. At this catch-up the bomb-debris kinetic energy adds to the energy of the outer shock, in effect increasing the yield driving the shock. After the first maximum the power falls rapidly until the temperature of the shock drops to about $0.8 \mathrm{eV}$. 
3. As the shock temperature continues to drop below $0.8 \mathrm{eV}$, the effective specific heat of the air decreases (equation of state of air), giving rise to a momentary increase in the shock temperature and an additional small maximum (or inflection) in the radiated power.

Also, the time of the power rise at the beginning of the first pulse can be strongly influenced by the so-called $x$-ray veil. The veil is a diffusely heated region outside the earlytime fireball which is produced during the initial bomb $x$-ray emission by the higher energy $\mathrm{x}$-rays whose mean free paths are larger than the fireball. If veil temperatures outside the fireball exceed about $0.7 \mathrm{eV}$, the veil hides the early evolution of the fireball. There is an abrupt increase in radiated power when the fireball "breaks through" the $\mathrm{x}$-ray veil $\left(t_{\text {veil }}\right.$ in Table IV). The $\mathrm{x}$-ray veil temperatures are a strong function of the device mass and other design details. The veil is generally not significant in small-yield or large-mass cases.

Since real nuclear bombs are rarely spherical, the detailed features in the first thermal pulse which depend on device mass, etc., can be expected to be smeared out in an actual fireball radiant power vs time signature, in contrast to the highly structured first pulse which we compute with the spherical RADFLO code. (Note that the present code is able to compute details in the first pulse which earlier versions of the code could not.) To investigate this point we ran a number of $2 \mathrm{kt}$ calculations using different values of bomb mass, as enumerated in Table IV. It can be noted that the computed first maximum time increases with increasing mass, and the fine structure disappears. An actual power-time curve might be expected to resemble an average of many RADFLO computed power-time curves with different device masses.

The reason for the departure of the $t_{\min }$ times from the scaling law for small yields below about $0.3 \mathrm{kt}$ seems to be related to the fact that the shock temperature at $t_{\min }$ increases with decreasing values of the yield, while the temperature at the time of the post-first-max decreasing-specific-heat blip decreases with decreasing yield, leading to a coincidence of the minimum time and the blip when the yield is about $0.2 \mathrm{kt}$. When the yield is $0.02 \mathrm{kt}$ the blip occurs after the minimum.

The main fireball radiative and hydrodynamic growth is not affected by nonequilibrium chemistry. Thus the timings of the various features in the power-time curves are not affected either. However, the radiated power magnitudes can be affected stronglyespecially at early times, and especially for large-yield cases. In the standard $1 \mathrm{Mt}$ case the chemistry reduces the height of the first maximum by a factor of five. 
As the shot altitude is increased, the time duration of the optical pulse decreases, as does the depth of the minimum. It is beyond the scope of this report to discuss bhangmeter scaling laws for high-altitude events, but they are discussed in Zinn, Kodis, and Sutherland (1974). Another high-altitude phenomenon (at ca $20 \mathrm{~km}$ ) seems to be a distinct splitting of the first maximum pulse into a velfb $=50 \mathrm{~km} / \mathrm{s}$ pulse and a debris-shock catch up pulse.

A few sample computed power-time plots are shown in Figures 8, 9, and 10.

Figure 8 shows a pair of power-time plots (done with HYCHEM and RADFLO respectively) for a $1 \mathrm{kt}$ bomb with a mass of $100 \mathrm{kgm}$ in sea level-density air. By our abovedescribed bomb-energy-partition model, the debris-surface radiating temperature came out to be only $1.2 \mathrm{eV}$, and essentially all of the bomb yield ended up as internal and kinetic energy (assumed 50-50), with less than .001 kt radiated away (i.e. no x-rays). The initial surface expansion velocity was about $260 \mathrm{~km} / \mathrm{s}$, and the debris-shock velocity was only slightly larger. At that velocity the debris shock drove a strong radiative precursor wave ahead of it, which moved faster than the shock itself, and whose effective radiating temperature was only about $2 \mathrm{eV}$-much less than the actual Rankine-Hugoniot temperature of the shock (Zinn and Sutherland 1981, 1982). As the shock expands outward its velocity decreases rapidly. By 15 microseconds it has dropped to about $70 \mathrm{~km} / \mathrm{s}$, at which point the separated precursor is beginning to form its own shock, and its brightness increases as the velocity decreases. Thus the fireball radiant power increases very rapidly, due to the combined effects of the increasing brightness and the increasing fireball surface area. At about 23 microseconds the power passes through a small maximum, when the shock velocity is $\mathbf{5 0}$ $\mathrm{km} / \mathrm{s}$ (the velocity at which the shock brightness is maximum). After that the power drops momentarily before continuing to rise. At about 44 microseconds the debris shock catches up with the separate shock from the precursor, causing a momentary surge in shock strength and a corresponding increase in brightness. After this time the shock velocity and the luminosity decrease rapidly. The first maximum (i.e. the maximum of luminosity $\times$ surface area) comes slightly after the shock catch up time. After first maximum both the power and the brightness decrease smoothly. (The small discontinuous drop at 106 microseconds is a numerical artifact arising from a logical switch to stop using the SSW patch at that point.) At about 1 millisecond the shock temperature has dropped to about $0.8 \mathrm{eV}$-the point at which the air-specific heat starts to fall rapidly. This results in a momentary temperature increase and a brief surge in power. After that time the shock strength continues to drop, as does its luminosity. But at the same time, the shock begins to become transparent to visible 


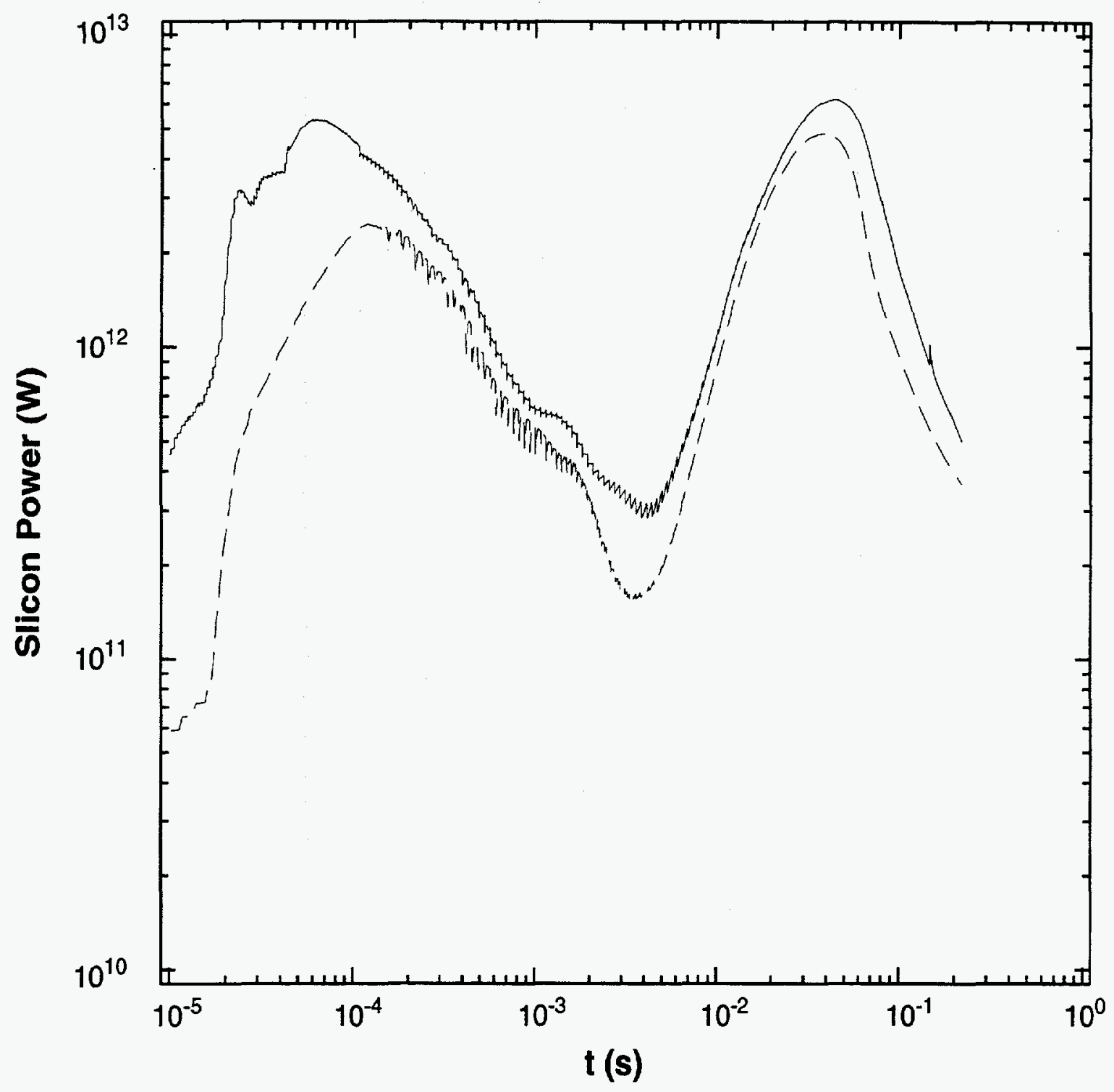

Figure 8. Computed radiant power (silicon band) vs time for a 1 kiloton explosion of a device with mass $100 \mathrm{kgm}$ in sea level-density air. HYCHEM (solid line) and $\mathrm{RADFLO}_{s}$ (dashed curve) results are shown. Both simulations used eight equally massed bomb cells with identical partitioning of $\mathrm{x}$-ray, kinetic, and internal energies. 
light (due to a drop in fractional ionization), and one begins to see the shock and the hotter air farther in as separate entities ("shock breakaway"). In this time regime there is a developing tradeoff between the decreasing brightness of the shock and its increasing transparency to the hotter and brighter air within, so that the radiant power, which has been decreasing, begins to increase again. Then we have passed through $t_{\min }$ (about 4.3 milliseconds).

During this same time period the temperatures in the fireball interior have been continually equilibrating by radiation transport (at far-UV wavelengths), leading to a structure approximating an "isothermal sphere." After $t_{\min }$ the fireball thermal (and optical) radiation comes mainly from this isothermal sphere, which continues to expand hydrodynamically and to cool. The rapidly increasing surface area causes the radiated power to increase for a while, but eventually, at $t_{2 \max }$, the effects of the combined radiative and hydrodynamic cooling become dominant and the radiated power starts to drop. In this time regime, as the fireball temperature drops so does its fractional ionization, so that the fireball becomes increasingly transparent and its emissivity drops. Eventually, after about $3 \times t_{2 \max }$, it becomes possible to see the more luminous debris-air cloud in the fireball interior. The debris is evident in the computed brightness profiles but does not have a noticeable effect on the power-time curves. In the RADFLO $\mathrm{S}_{\mathrm{S}}$ computation the minimum and second maximum times are slightly earlier than in the HYCHEM run, while the first maximum time is considerably later. The first maximum power is also quite a bit lower.

Figure 9 shows a pair of bhangmeter power-time curves for a $1 \mathrm{Mt}$ bomb with a mass of $1000 \mathrm{kgm}$ in sea level-density air. Some of the details are quite different from the 1 $\mathrm{kt}$ results. In this case our bomb energy partition model leads to a debris radiating temperature greater than 2 kilovolts, and $86.6 \%$ of the yield is radiated away as x-rays, leaving only 67 $k t$ each in kinetic and internal energy. The debris-surface expansion velocity is about 1500 $\mathrm{km} / \mathrm{s}$. The large $\mathrm{x}$-ray yield and high-radiating temperature lead to a very pronounced $\mathrm{x}$-ray veil. The visual radius of the veil is about 56 meters and its luminosity is fairly low while it completely obscures the smaller and brighter fireball within. The early fireball, produced during the $\mathrm{x}$-ray deposition, is a fairly isothermal sphere with a temperature of about $100 \mathrm{eV}$ and a radius of about 40 meters. For the next 60 microseconds or so, the fireball expands rapidly by radiation transport, but its expansion velocity drops rapidly as the fireball engulfs more air and cools. By 60 microseconds the velocity has dropped to a point where hydrodynamic motions can compete and a shock forms at the front. The subsequent expansion proceeds mostly by hydrodynamics. However, none of these details are visible from the 


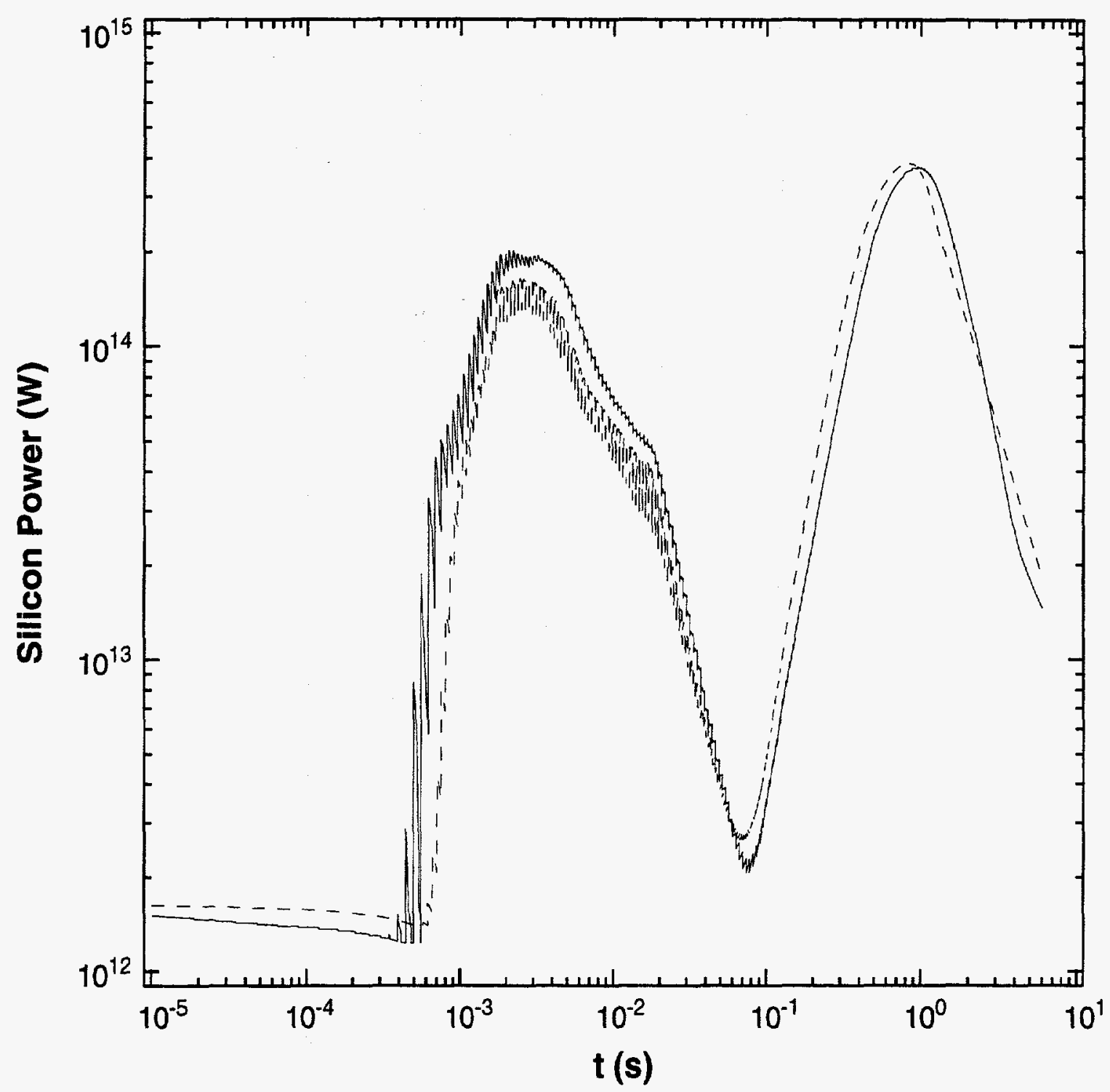

Figure 9. Computed radiant power (silicon band) vs time for a 1 Megaton explosion of a device wiht mass $1000 \mathrm{kgm}$ in sea level-density air. HYCHEM (solid curve) and RADFLO (dashed curve) results are shown. Again, both simulations used eight bomb cells with identical partitioning of $\mathrm{x}$-ray, kinetic, and internal energies. 
outside because they occur inside the $x$-ray veil. The fireball breaks through the veil at about 300 microseconds, giving rise to a sharp increase in radiated power. The debris shock does not catch up with the outer fireball shock until about 1.5 milliseconds, by which time it is quite weak and produces only a minimal effect on the brightness. The first maximum occurs shortly after the debris-shock catch up. From this point on the fireball expansion phenomenology is qualitatively similar to the $1 \mathrm{kt}$ case. The RADFLO and HYCHEM curves are quite similar in this $1 \mathrm{Mt}$ case, although again the RADFLO $\mathrm{S}_{\mathrm{S}}$ minimum and second maximum times are slightly earlier than the corresponding HYCHEM times. The veil breakthrough time is slightly later in the RADFLOS run.

We have also run $1 \mathrm{Mt}$ cases with larger bomb masses. With a mass of $4000 \mathrm{kgm}$ the $\mathrm{x}$-ray temperature is lower than in the $1000 \mathrm{kgm}$ case and the $\mathrm{x}$-ray yield fraction is reduced to $60.7 \%$, with the result that the $x$-ray veil is much less pronounced. The veil breakthrough time is reduced to 30 microseconds.

We have also run a $1 \mathrm{Mt}$ sea level case with a mass of $1000 \mathrm{kgm}$ with inclusion of chemistry. In this case the times of all features in the power-time signal were the same as those in Fig. 9, but the height of the first maximum was reduced by a factor of five, and the height of the second maximum was reduced by a factor of two. The height of the minimum was unchanged.

To illustrate the grossly different structure of bhangmeter signals for high-altitude events, we show in Figure 10 a computed power-time curve for a $100 \mathrm{kt}$ shot at $50 \mathrm{~km}$

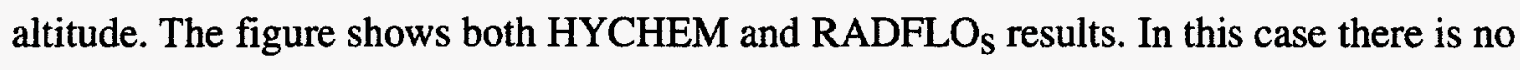
minimum. At this altitude hydrodynamics plays a minor role compared to radiation transport. The fireball becomes transparent and the debris cloud becomes visible at $0.015 \mathrm{~s}$. The debris radiation does not materially affect the radiant power vs time curves. The final radiated yield at $0.1 \mathrm{~s}$ is $34 \%$ of the original $100 \mathrm{kt}$ in the HYCHEM simulation.

Another useful fireball observable is its radius vs time evolution. Before the Trinity test in early 1945 it was predicted by Bethe et al. (1947) that the early fireball growth should be approximated by a similarity solution, known currently as the Taylor-Sedov blast wave. The blast-wave model is based on the assumptions that gamma (the heat-capacity ratio) is a constant and that no appreciable energy is lost by radiation, and it leads to the expectation that the fireball radius should increase as the $2 / 5$ power of the time (see e.g. Bethe 1964. and Zel'dovich and Raizer 1967). It is interesting to compare it with RADFLOcomputed radius vs time results. 


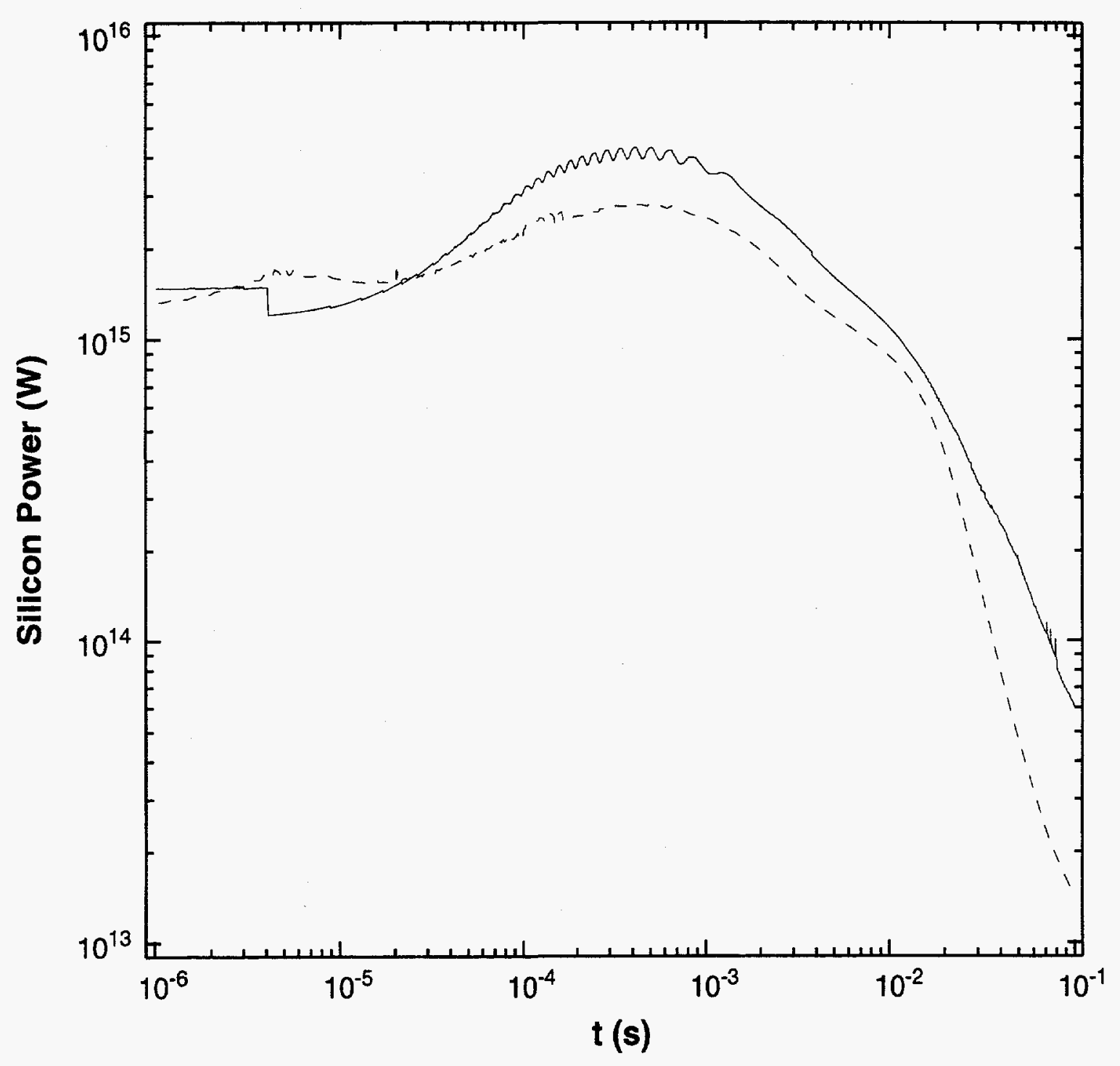

Figure 10. Computed radiant power in the silicon band vs time for a 100 kiloton explosion of a device with mass $460 \mathrm{kgm}$ in air at $50 \mathrm{~km}$ altitude. The

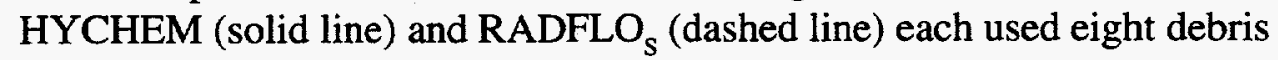
cells, Gudunov hydrodynamics, and identical initial energy partitioning. 
A plot of the computed fireball radius vs time for $1 \mathrm{kt}, 20 \mathrm{kt}$, and $1 \mathrm{Mt}$ cases are shown in Figure 11. The curves are by no means straight lines on the log-log scales. However consider the $1 \mathrm{kt}$ case, between the time of debris-shock catch up (when for the first time the full yield is communicated to the outer shock) and the time of the $0.8 \mathrm{eV}$ blip (after which gamma is increasing rapidly), there is a linear segment that corresponds to a power law $R_{f b}=$ const $\times t^{0.36}$-not too different from the $2 / 5$ power. The $20 \mathrm{kt}$ and $1 \mathrm{Mt}$ cases have similar slopes for the linear part of the log-log plot. Note that the quantity plotted is the visual fireball radius, defined as the locus of optical depth $=1$. Hence the collapse to zero as the fireball becomes transparent.

We believe that the current HYCHEM model represents a significant improvement over earlier RADFLO versions, but some persistent weak points are discussed in Appendix B.

\subsection{SUMMARY}

This report has described the RADFLO family of computer codes for the modeling of nuclear fireballs, including their general characteristics and a historical discussion of how they have evolved since the original RADFLO code of 1963. We have described the similarities and differences between the current LANL codes RADFLO $_{Z}$ and HYCHEM

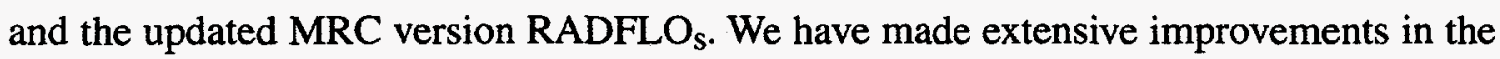
codes during the past few years, including (1) replacement of the original Richtmyer-von Neumann hydrodynamics algorithm with a one-dimensional version of the modern Godunov Riemann-solver algorithm, (2) extension of our steady-state shock and thermal wave (SSW) computations to include lower ambient air densities for high-altitude applications, and (3) improvements in the method of applying the SSW patch correction for computing fireball luminosity and radiant power at early times. We have described these new developments in some detail, including a brief discussion of non-equilibrium chemistry effects and their computation in HYCHEM.

We have also made a large number of test computations with the improved codes. The results are described, including scaling laws for the times of first maximum, minimum, and second maximum as functions of the yield, and variations due to bomb mass, shot altitude, and departures from chemical equilibrium. For a few typical cases we have included a qualitative description of the phenomenology and growth of the fireball and observables such as radiant power vs time and radius vs time. Some comparisons between $\mathrm{RADFLO}_{\mathrm{z}}$ and $\mathrm{RADFLO}_{\mathrm{s}}$ results are also included. 


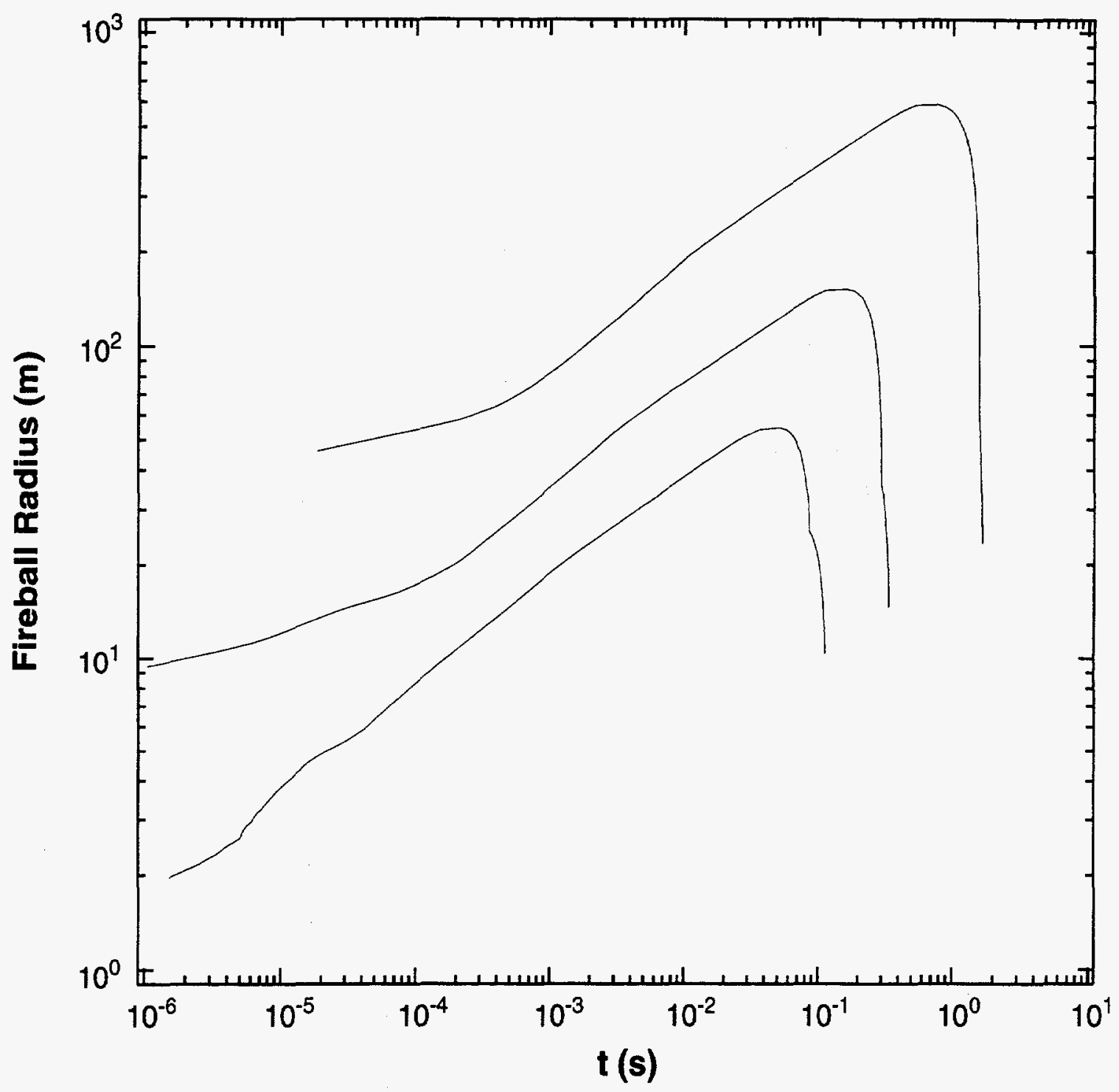

Figure 11. Computed fireball radius vs time for a 1 kiloton, 20 kiloton, and 1 Megaton explosions, from botton to top respectively. The default parameters were used for producing bomb masses of 100,270 , and $1000 \mathrm{kgm}$, respectively, in eight debris cells. The simulations were done using HYCHEM and Godunov hydro. This is the visual fireball radius, defined as the locus of optical depth $=1$. Therefore the fireball radius collapses to zero as the fireball becomes transparent. 


\section{Appendix A: SSW Computations}

To extend the range of HYCHEM to altitudes above sea level a set of SSW computations were run covering an extended range of ambient air densities. The range now extends from downward to . These computations were based on the same algorithms and same computer code as described in Zinn and Sutherland (1981). The results are summarized in Figure 12, which shows computed values of effective radiating temperature for visible light plotted vs the front propagation velocity, for six different values of the background air density.

\section{Appendix B: Numerical Artifacts}

The use of a finite-difference grid requires that material velocities and state variables are averaged over grid cells as an approximation to physical reality. It is hoped that the approximation improves as cell dimensions are reduced. We have done a large number of numerical experiments to examine this point, but are always limited by available computer time, since the running time is proportional to the -2 power of the cell thickness (approximately). At all levels of cell fineness we see some details in the computed fireball front velocity that we do not fully understand, and these details lead to corresponding variations in fireball brightness and power. The velocity is computed by numerical differentiation of the fireball radius vs time-an inherently noisy process. A bothersome question is how well or badly do we compute the process of transition between an expanding thermal-wave front and the shock wave that develops from it. That process evolves on a spatial scale much smaller than our mesh size, so that we can not resolve it. However it occurs when the front velocity is in the range 70 to $100 \mathrm{~km} / \mathrm{s}$, when the brightness is relatively low but increasing and the power is on the steep rise toward first maximum (unless the fireball is still obscured by the $\mathrm{x}$-ray veil). Therefore the details of the radiative expansion to shock-wave transition do not affect the power-time curves very much.

We have already described our procedure for patching the separately computed SSW brightness results into the radiation transport computation when the front velocity exceeds a threshold value vquitfit, which is usually set at $30 \mathrm{~km} / \mathrm{s}$. We discontinue implementation of the patch when the velocity drops below that value, and use the standard RADFLO radiation transport algorithm to compute the brightness. In some cases this change in the algorithm results in a small discontinuous drop or jump up in the computed power. Figure 8 shows such a discontinuity at $1.06 \times 10^{-4} \mathrm{~s}$. 


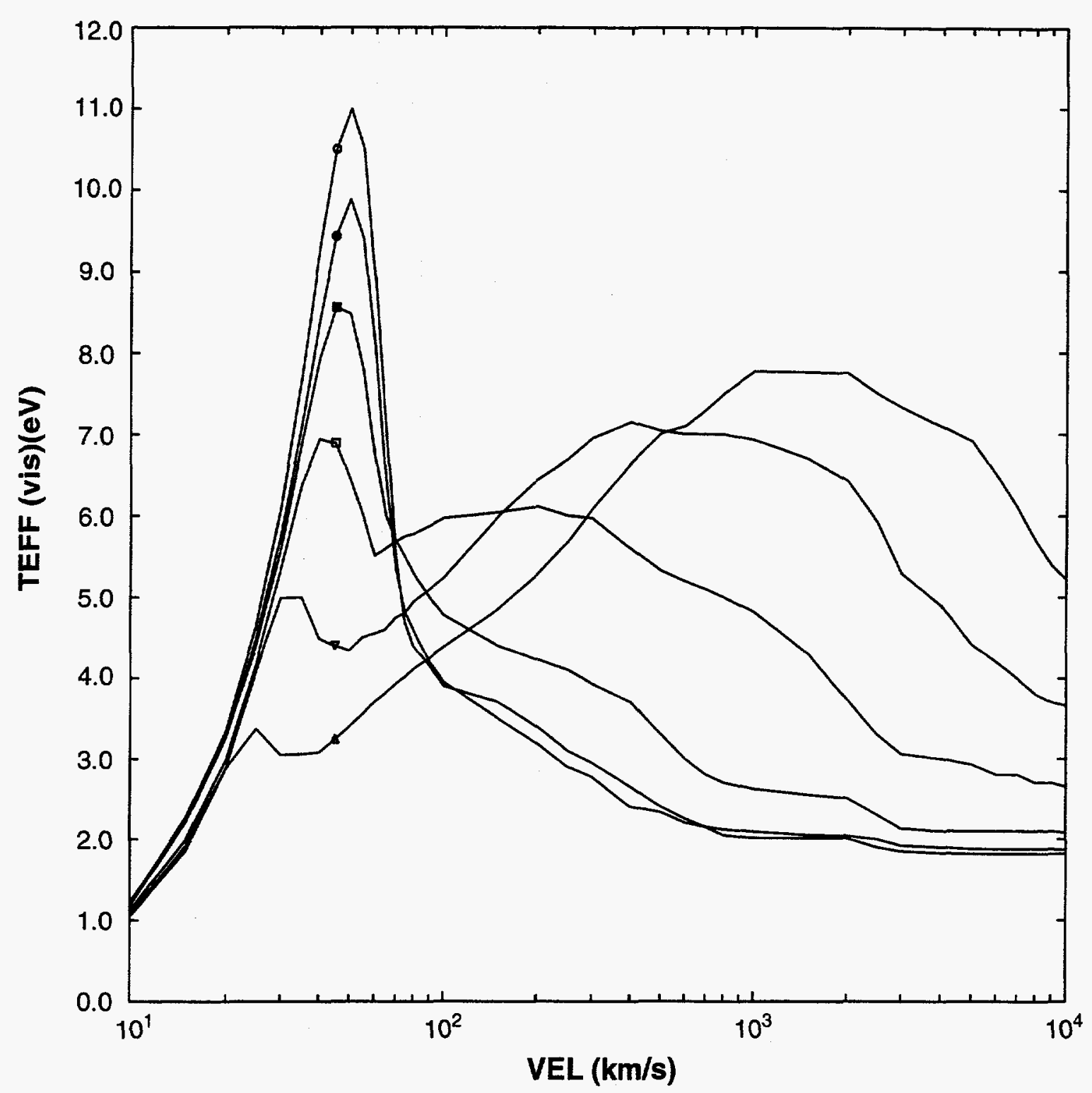

Figure 12. Plots of effective radiating temperature (for visible light) vs the wavefront propagation velocity for six different values of the background air density. (Open circle-density $=0.00123 \mathrm{~g} / \mathrm{cm}^{3}$; solid circle-density $=0.0001$; solid square-density $=0.0005$; open square-density $=0.0002$; down triangle-density $=0.0001$; up triangle - density $=5 \times 10^{-5}$.) 
An area of much-needed improvement in the HYCHEM model is the treatment of gamma rays and neutrons. They are approximated in the current model by a pair of analytic expressions, which may be wrong in some cases by an order of magnitude. Given today's easily accessible computational power, deposition via a real radiation transport algorithm seems feasible. We believe this would vastly improve the pre-first-maximum part of the power-time curve. Second, the bomb debris could be modelled as a second turbulent fluid because of the debris/air interface, Taylor unstable nature. The influence of bomb debris on the free electron density and the total opacity could then be directly calculated.

\section{Acknowledgments}

We gratefully acknowledge a number of useful discussions, over a number of years with E. M. Jones, J. Kodis, Henry Horak, D. S. Sappenfield, T. H. McCartor, F. Fajen, and D. Landman. We especially thank P. A. Medvick for a critical reading of this manuscript. This work was supported in part by the Department of Energy.

\section{References}

Addessio, F. L., J. R. Baumgartner, J. K. Dukowicz, N. L. Johnson, B. A. Kashiwa, R. M. Rauenzahn, and C. Zemach, "CAVEAT: a Computer Code for Fluid Dynamics Problems with Large Distortion and Internal Slip," Los Alamos National Laboratory report LA-10613MS, Rev. 1. (U).

Allen, R. A., J. C. Camm, and J. C. Keck, "Radiation from Hot Nitrogen," AVCO Corporation Research Report 102, April 1962 (U).

Armstrong, B. H., D. H. Holland, and R. E. Meyerott, "Absorption Coefficients of Air from 22,000 to 220,000 K," Lockheed Aircraft Corp, Air Force Special Weapons Center, AFSWCTR-58-36, August 1958 (U).

Bethe, H. A., K. Fuchs, J. O. Hirschfelder, J. L. Magee, R. E. Pierls, and J. von Neumann, "Blast Wave," LA-2000, August 1947 (U).

Bethe, H. A., "Theory of the Fireball," Los Alamos LA-3064, February 1964 (U).

Breene, R. G., Jr., "Molecular Spectral Line Broadening Study," Air Force Weapons Laboratory, AFWL-TR-66-35, August 1966 (U).

Buttrey D. E. and H. R. McChesney, "Opacity of Low Temperature Air," Lockheed Missiles and Space Company, AFWL-TR-65-134, November 1965 (U). 
Buttrey, D. E., H. R. McChesney, and L. A. Hooker, "Broadening of Spectral Lines of Air Molecules,” Air Force Weapons Laboratory, AFWL-TR-66-144, May 1967 (U).

Camm, J. C., B. Kivel, R. L. Taylor, et al., "Opacity of Low-Temperature Air," AVCO Everett Research Laboratory, AFWL-TR-66-127, March 1967 (U).

Churchill, D. R., S. A. Hagstrom, and R. K. M. Landshoff, "The Spectral Absorption Coefficient of Heated Air," J. Quant. Spectrosc. Radiat. Transfer, 4, 291-321, 1964.

Churchill, D. R. and S. A. Hagstrom, "Infrared Absorption in Heated Air from VibrationRotation Bands of Nitric Oxide," Lockheed Missiles and Space Co. report 2-12-64-2, December 1964 (U).

Churchill, D. R., B. H. Armstrong, R. R. Johnston, and K. G. Mueller, "Absorption Coefficients of Heated Air: a Tabulation to $24000 \mathrm{~K}$," J. Quant. Spectrosc. Radiat. Transfer, 6, 371-442, 1966.

Churchill, D. R., S. A. Hagstrom, J. D. Weisner, and B. H. Armstrong, "The Spectral Absorption Coefficient of Heated Air," Lockheed Missiles and Space Co. report 2-57-62-2, November 1962 (U).

Cox, A. N. and J. N. Stewart, "Radiative and Conductive Opacities and Monochromatic Absorption Coefficients for Air," Los Alamos Scientific Laboratory report LAMS-3014, 1964 (U).

Dukowicz, J. K., "A General, Non-Iterative Riemann Solver for Godunov's Method," J. Comput. Phys., 61, 119-137, 1985.

Fuchs, K., G. J. Kynch, and R. Peierls, "The Equation of State of Air at High Temperatures," British Department of Atomic Energy, BDDA-16, Report MS 61, December 1942. (U.S. Dept. of Commerce, PBL 87018).

Generosa, J. and R. Harris, 1970, J. Chem. Phys. 53: 3174.

Gilmore, F. R., "Equilibrium Composition and Thermodynamic Properties of Air to 24000 K," RAND corporation report RM-1543, August 1955 (U).

Gilmore, F. R., "Equilibrium Composition and Thermodynamic Properties of High Temperature Air," RAND corporation report RM-1543, December 1958 (U).

Gilmore, F. R. and A. L. Latter, "Approximate Thermodynamic and Radiation Properties of Air Between 2 and 600 Volts," RAND research memorandum RM-1617, January 1956 (U). 
Harris, R. A., M. Blackledge, and J. Generosa, 1969, J. Mol. Spectrosc. 30: 506.

Harris, R. A. and L. Sullo, "ABSCO, A Computer Program which Calculates and Displays Locally Integrated Planck Means," Air Force Weapons Laboratory report SYT TN 70-17, 1970 (U).

Hilsenrath, J., M. G. Green, and C. W. Beckett, "Thermodynamic Properties of Highly Ionized Air," AFSWC Report SWC-TR-56-35, 1956 (U).

Hilsenrath, J., M. Klein, and H. W. Woolley, "Tables of Thermodynamic Properties of Air Including Dissociation and Ionization from $1500 \mathrm{~K}$ to $15000 \mathrm{~K}$," NBS Report AEDC-TR59-20, $1959(\mathrm{U})$.

Horak, H., "Vela Event Alert 747," Los Alamos National Laboratory report LA-8364-MS, 1980 (SRD).

Horak, H., and J. Kodis, "RADFLO-A User's Manual," Los Alamos National Laboratory report LA-9245-MS, 1983 (U).

Horak, H. G., D. S. Sappenfield, J. W. Kodis, C.D. Sutherland, and J.Zinn, "Comparisons of RADFLO Calculations with Observed Data for Three Nuclear Explosions," Los Alamos National Laboratory report LA-9832-MS, July 1983 (SRD).

Jones, E. M. and John W. Kodis, "Rayleigh-Taylor Instabilities at the Debris/Air Interface," Los Alamos National Laboratory report LA-10823-MS, 1986 (U).

Jones, E. M., B. W. Smith, W.C. Straka, J.W. Kodis, and H. Guitar, "Interacting Supernova Remnants: Tunnels in the Sky," 1979, Ap. J., 232, 129.

Jones, E. M., B. W. Smith, and W. C. Straka, "The Formation of Supernova Remnants: The Pre-Blast-Wave Phase," 1981, Ap. J., 249: 185-194.

Jones, E. M., R. W. Whitaker, and J. W. Kodis, “Concentric Nuclear Explosions," Los Alamos National Laboratory report LA-9688-MS, 1983 (U).

Jones, E. M., J. Childers, and J. W. Kodis, "Geometry of Fireball Spikes: Priscilla and Socorro," Los Alamos National Laboratory report LA-10900-MS, 1982 (U).

Jones, E. M., R. W. Whitaker, H. G. Horak, and J. W. Kodis, "Low-Yield Nuclear Explosion Calculations: The 9/22/79 VELA Signal (U)," Los Alamos National Laboratory report LA9062, 1982 (SRD). 
Johnston, R. R., R. W. Hillendahl, R. A. Berg, R. K. Landshoff, and O. R. Platas, "Optical Modelling," Lockheed Missile and Space Corp., LMSC B303615, 1971 (SRD).

Johnston, R. R. and C. R. Platas, "Atomic Line Transitions and Radiation from High Temperature Air," Lockheed Missiles and Space Company Technical Report N-3L-69-1, July 1969 (U).

Keck, J. C., R. A. Allen, and R. L. Taylor, "Electronic Transition Moments for Air Molecules," AVCO Corporation Research Report 149, March 1963 (U).

Keck, C. K., J. C. Camm, B. Kivel, and T. Wentink, "Radiation from Hot Air," Annals of Physics, 7, 1-38, 1959.

Kivel, B. and K. Bailey, "Tables of Radiation from High Temperature Air," AVCO Research Laboratory Report 21, December 1957 (U).

Knapp, W. S., R. J. Jordano, J. H. Thompson, and K. Schwartz, "Weapon Output, Energy Deposition, and Atmospheric Chemistry Models for ROSCOE," General Electric TEMPO, GE74TMP-59, 1974 (U) (SRD-CNWDI).

Latter, A., "Equation of State of Air on the Statistical Model," RAND Corporation research memorandum RM-1344-AEC, September 1954 (U).

Mayer, H., "Methods of Opacity Calculations," Los Alamos Scientific Laboratory report LA-647, October 1947.

Mitchell, C. K., "Analysis of the Flathead and Navajo Chord Experiments," EGG-1183063, 1979, (U) (SRD).

Mueller, K. G., "The Absorption Coefficient of $\mathrm{NO}_{2}$," Lockheed Missiles and Space Co. report 1-27-63-1 (Defense Atomic Support Agency 1416), August 1963 (U).

Patch, R. W., W. L. Shackleford, and S. S. Penner, "Approximate Spectral Absorption Coefficient Calculations for Electronic Band Systems Belonging to the Diatomic Molecules," J. Quant. Spectrosc. Radiat. Transfer, 2, 263-271, 1962.

Sappenfield, D., "Early-time Optical Studies," Mission Research Corporation report MRCR67, November 1973 (SRD).

Sappenfield, D., "Early-time Optical Studies II," Mission Research Corporation report MRCIR74217, August 1974 (SRD). 
Sappenfield, D., "Early-time Optical Studies III," Mission Research Corporation report MRCR178, May 1975 (SRD).

Sappenfield, D., "Early-time Optical Studies IV," Mission Research Corporation report MRCR178, September 1976 (SRD).

Sappenfield, D., "Close-in Thermal and Hydrodynamic Effects of Low-Yield Nuclear Explosions," Los Alamos National Laboratory report LA-9926-MS, October 1983 (SRD).

Sappenfield, D., "Additional Comparisons of Data from Low-Altitude Nuclear Tests with RADFLO Calculations," Los Alamos National Laboratory report LA-10088-MS, 1984 (SRD).

Sappenfield, D., "Improved Optical Detection/Diagnostics for a Nuclear Explosion Below 10 km Altitude," Los Alamos National Laboratory report LA-10427-MS, June 1985 (SRD).

Skumanich, A., "Teak Fireball Formation, Radiative Growth and Brightness History," Los Alamos Scientific Laboratory report LA-MS- 2453, August 1960 (SRD).

Sowle, D. H., P. G. Fischer, and D. S. Sappenfield, "An All-Altitude Air Burst Code, Volume I: New Techniques," MRC-R-21, 1972 (U).

Symbalisty, E. M. D., "Some NUDET Effects Due to Water Containment," Los Alamos National Laboratory report LA-12775-MS, 1994 (U).

Symbalisty, E. M. D., "Fast and Accurate Shock Envelope Determination," Los Alamos National Laboratory report LA-11999-MS, 1991 (U).

Symbalisty, E. M. D., D. S. Sappenfield, C. D. Sutherland, and B. A. Kashiwa, "Physics and Chemistry of Late-Time Fireball Simulations without a Geomagnetic Field," Los Alamos National Laboratory report LA-11122-MS, 1987 (U).

Taylor, R. L., "Continuum Infrared Spectrum of High Temperature Air," AVCO Corporation Research Report 88, June 1960 (U).

Taylor, R. L. and G. Caledonia, "Experimental Determination of the Cross Section for Neutral Bremsstrahlen II. High Temperature Air Species O, N, and $\mathrm{N}_{2}$," AVCO Corporation Research Report 312, November 1968 (U).

Von Neumann, J. and R. D. Richtmyer, "A Method for the Numerical Calculation of Shocks," J. Appl. Phys., 21: 232-237, 1950. 
Wentink, T., E. P. Marram, and L. Isaacson, "Research on the Opacity of Low Temperature Air: Oscillator Strengths and Transition Moments of Molecular Band Systems," AVCO Corporation, AFWL-TR-67-6, July 1967 (U).

Woolley, H. W., "The Calculation of Thermodynamic Properties of Gases at High Temperatures," Air Force Special Weapons Center report SWC-TDR-62-21, March 1962 (U).

Wray, K. L. and R. L. Taylor, "Four-Micron Band Radiation from High Temperature Air," AVCO Corporation Research Report 322, January 1969 (U).

Zel'dovich, Ya. B. and Yu. P. Raizer, Physics of Shock Waves and High-Temperature Hydrodynamic Phenomena, Academic Press, New York and London, 1967.

Zinn, J., "Radiation Transport in Bluegill," Los Alamos National Laboratory report LA3095-MS, June 1964 (SRD).

Zinn, J. and F. E. Fajen, "A Radiation Transport and Hydrodynamic Computation of Fireball Growth in Bluegill," Journal of Missile Defense Research, JMDR-65-2, Vol. II, Winter 1964 (SRD).

Zinn, J., "Fireball Behavior in Bluegill and a Hypothetical 100-Megaton Explosion at Bluegill Altitude," Los Alamos National Laboratory report LA-3239-MS, January 1965 (SRD).

Zinn, J., T. R. Connor, and D. S. Sappenfield, "Foreign Test Diagnostics. Study of Early Fireball Expansion Phenomenology," Los Alamos National Laboratory report LA-4408MS, March 1970 (SRD).

Zinn, J., J. W. Kodis, M. T. Sandford II, H. Hoerlin, and H. G. Horak, "Radiation Hydrodynamics Computations for Ten Sealevel Test Events," Los Alamos National Laboratory report LA-4995, September 1972 (SRD).

Zinn, J., "A Finite Difference Scheme for Time-Dependent Spherical RadiationHydrodynamics Problems,” J. Comput. Phys., 13, 569 (1973).

Zinn, J. and R. C. Anderson, "Structure and Luminosity of Strong Shock Waves in Air," Phys. Fluids, 16, 19-63 (October 1973).

Zinn, J., J. W. Kodis, and C. D. Sutherland, "Status Report on Fireball Radiation Hydrodynamics Computations," Los Alamos National Laboratory report LA-5591-SR, May 1974 (SRD). 
Zinn, J., P. J. Bottoms, M. S. Tierney, C. D. Sutherland, and E. Jones, "Microwave Radiometry Measurements in Operation Alondra," Los Alamos National Laboratory report LA-5657MS, June 1974 (SRD).

Zinn, J., C. D. Sutherland, H. Hoerlin, C. D. Wilson, and C. K. Mitchell, "Chemistry of a Nuclear Fireball," Los Alamos National Laboratory report LA-8391-MS, June 1980 (SRD).

Zinn, J. and C. D. Sutherland, "Structure and Luminosity of Radiative Thermal Waves in Air," Los Alamos National Laboratory publication LA-UR-81-1893, June 1981 (U).

Zinn, J., C. D. Sutherland, and C.K. Mitchell, "Chemistry and Spectroscopy of a Fireball," Los Alamos National Laboratory report LA-9551-MS, October 1982 (U).

Zinn, J. and C. D. Sutherland, "Special Numerics for a Nuclear Fireball Model," Los Alamos National Laboratory report LA-9413-MS, June 1982 (U).

Zinn, J. and C. D. Sutherland, “An Analysis of Sealevel Teller Light," Los Alamos National Laboratory report LA-11022, 1987 (SRD). 